\title{
Higher-Harmonic-Control Algorithm for Helicopter Vibration Reduction Revisited
}

\author{
Dan Patt, ${ }^{*}$ Li Liu, ${ }^{*}$ Jaganath Chandrasekar, ${ }^{\dagger}$ Dennis S. Bernstein, ${ }^{\dagger}$ and Peretz P. Friedmann ${ }^{\S}$ \\ University of Michigan, Ann Arbor, Michigan 48109-2140
}

\begin{abstract}
The higher-harmonic-control (HHC) algorithm is examined from a control theory perspective. A brief review of the history and variants of HHC is given, followed by a careful development of the algorithm. An analytic convergence and robustness analysis is then performed. Online identification with the adaptive variant of the algorithm is also addressed. A new version of the algorithm, relaxed HHC, is introduced and shown to have beneficial robustness properties. Some numerical results comparing these variants of the HHC algorithm applied to helicopter vibration reduction are also presented. The results presented unify and extend previous work on the higher-harmonic-control algorithm.
\end{abstract}

\section{Nomenclature}

$A$ between updates

$\boldsymbol{A}^{\star} \quad=$ adjoint matrix of $\boldsymbol{A},(\overline{\boldsymbol{A}})^{T}$

$\boldsymbol{D} \quad=$ matrix defined to be $\boldsymbol{T}^{T} \boldsymbol{Q T}+\boldsymbol{R}$

$F_{f} \quad=$ exponential window

$\boldsymbol{I}_{n} \quad=$ identity matrix of size $n \times n$

$J \quad=$ quadratic-form cost function

$J_{\mathrm{LS}, \infty}=$ converged quadratic-form cost function for adaptive control

$J_{T, \alpha, \infty}=$ converged cost function for relaxed invariant control

$J_{\hat{T}, \infty} \quad=$ converged cost function for invariant control with estimate $\hat{\boldsymbol{T}}$ for $\boldsymbol{T}$

$\boldsymbol{K}_{k+1}=$ matrix defined to be $\Delta \boldsymbol{u}_{k+1}^{T} \boldsymbol{P}_{k+1}$

$k=$ integer index between control inputs

$\boldsymbol{M} \quad=$ matrix defined to be $\boldsymbol{D}^{-1}\left(\boldsymbol{T}^{T} \boldsymbol{Q}+\boldsymbol{S}^{T}\right)$

$m=$ number of harmonics in control input

$N_{b} \quad=$ number of rotor blades

$\boldsymbol{P}_{k} \quad=$ matrix defined as $\left(\Delta \boldsymbol{U}_{k} \Delta \boldsymbol{U}_{k}^{T}\right)^{-1}$

$p \quad=$ number of harmonics in measured output

$Q \quad=$ weighting matrix for plant output in cost function of size $2 p \times 2 p$

$\boldsymbol{R}=$ positive-definite weighting matrix for control input in cost function of size $2 m \times 2 m$

rank $=$ matrix rank

$/ \mathrm{rev}=$ frequency of rotor revolution

$S \quad=$ cross-weighting term in objective function $J$

$\operatorname{spec}(\boldsymbol{A})=\operatorname{spectrum}$ of $\boldsymbol{A}, \operatorname{spec}(\boldsymbol{A})=\left\{\lambda_{A_{1}}, \ldots, \lambda_{A_{n}}\right\}$

$\boldsymbol{T}=$ sensitivity matrix relating control input to plant output

$\hat{\boldsymbol{T}} \quad=$ estimate of the sensitivity matrix $\boldsymbol{T}$

Presented as Paper 2004-1948 at the AIAA/ASME/ASCE/AHS/ASC 45th Structures, Structural Dynamics, and Materials Conference, Palm Springs, CA, 19-22 April 2004; received 27 May 2004; revision received 20 December 2004; accepted for publication 21 December 2004. Copyright (C) 2005 by the authors. Published by the American Institute of Aeronautics and Astronautics, Inc., with permission. Copies of this paper may be made for personal or internal use, on condition that the copier pay the $\$ 10.00$ per-copy fee to the Copyright Clearance Center, Inc., 222 Rosewood Drive, Danvers, MA 01923; include the code 0731-5090/05 \$10.00 in correspondence with the CCC.

*Postdoctoral Scholar, Department of Aerospace Engineering, 1320 Beal Avenue. Member AIAA.

${ }^{\dagger}$ Ph.D. Candidate, Department of Aerospace Engineering, 1320 Beal Avenue. Student Member AIAA.

${ }^{\ddagger}$ Professor, Department of Aerospace Engineering, 1320 Beal Avenue. Member AIAA.

${ }^{\S}$ François-Xavier Bagnoud Professor, Department of Aerospace Engineering, 1320 Beal Avenue. Fellow AIAA.

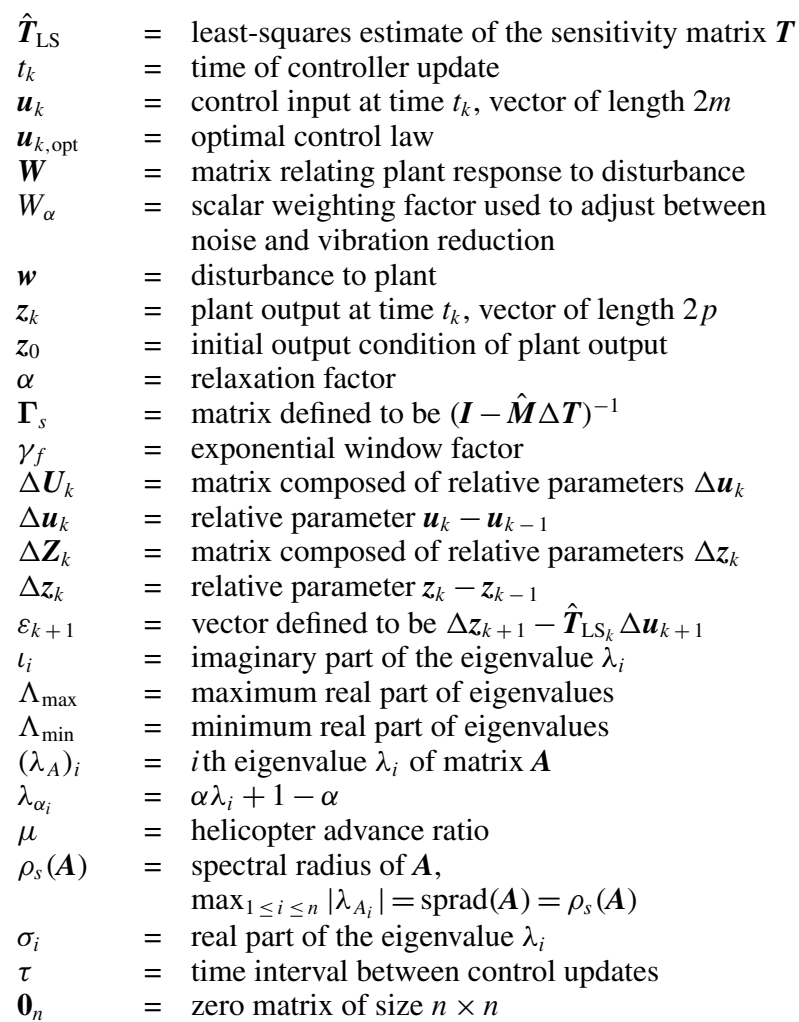

\section{Introduction}

Q TRINGENT requirements for low vibration levels (less than $0.05 \mathrm{~g}$ ) have motivated the development of active control concepts for vibration reduction. The various active control approaches that have been developed are described in Ref. 1. These control techniques fall into two separate categories: 1) active control approaches aimed at reducing vibrations in the rotor before they propagate into the fuselage and 2) active control approaches implemented in the fuselage using an approach known as active control of structural response (ACSR). Within the first category of active control, where the primary objective is to reduce vibrations in the rotor, two approaches have emerged. These are 1) higher harmonic control (HHC), where the blades are activated in the nonrotating swashplate by introducing pitch commands, and 2) individual blade control (IBC) where each blade can be controlled independently in the rotating frame. Several implementations of IBC are available: 1) the conventional or earliest implementation based on pitch actuation at the blade root in the rotating system, 2) actively controlled partial-span trailing-edge flaps, and 3) the active-twist rotor where the entire blade is twisted 


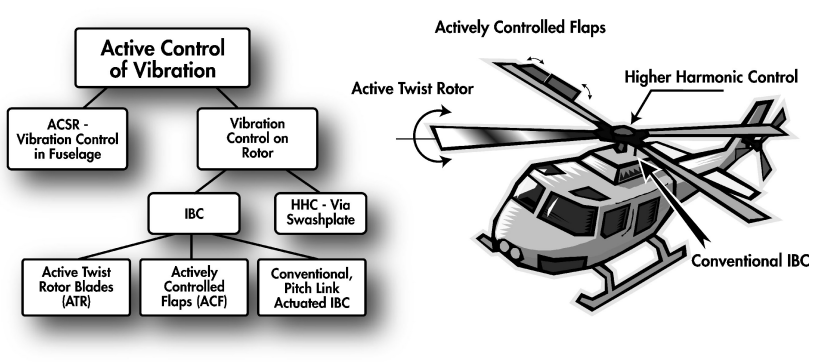

Fig. 1 Overview of active control techniques.

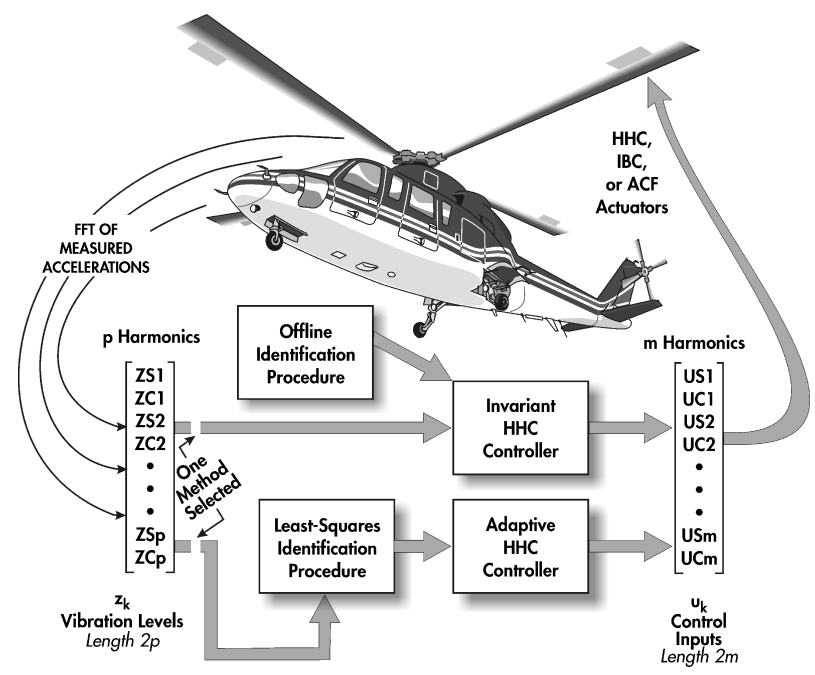

Fig. 2 Overview of HHC for vibration reduction in rotorcraft.

by piezoelectric fibers embedded in the blade. These approaches are illustrated schematically in Fig. 1, and additional description of these approaches can be found in Ref. 1. Both HHC as well as IBC active control approaches rely on what is known as the conventional HHC algorithm. Furthermore, even the implementation of ACSR is based on the HHC algorithm.

One of the most comprehensive overviews of the HHC algorithm and its variants was written by Johnson. ${ }^{2}$ This detailed report provided a history of the development of the algorithm and its implementation in early experimental and numerical studies. A graphical description of the algorithm and its implementation on a helicopter is shown in Fig. 2.

Johnson characterizes the variants of the HHC algorithm as sharing three properties:

1) The first property is a linear, quasi-static, frequency-domain model of the helicopter response.

2) The model is identified by a least-squared error or Kalmanfilter method. Identification can either be offline (invariant HHC) or online (adaptive HHC).

3) A quadratic-form cost function is used.

Since this review, ${ }^{2}$ a considerable amount of research on the HHC algorithm has been done, and its application has been extended beyond swashplate-actuated HHC systems to the IBC and ACSR approaches as mentioned earlier. Recent research has largely focused on identification techniques, application of the controller in both open-loop and feedback forms, and the effects of periodic coefficients and nonlinearity that are inherent to rotorcraft.

Of particular note among the many experimental studies performed with this algorithm is the work of Shaw and colleagues on the helicopter vibration problem, extending from the 1970s to the late 1980 s. $^{3-5}$ Initial open-loop wind-tunnel tests up to $\mu=0.3$ indicated that vibratory hub loads were essentially linearly dependent on the harmonic control input, confirming the feasibility of HHC for this application. This was followed by tests in closed-loop using real-time identification of the transfer function. In these studies, three different versions of HHC were considered, each using a different identification procedure: 1) a fixed-gain controller using local linearization of the current vibration levels, 2) a scheduled-gain controller that automatically selected the transfer function matrix based on the current flight condition, and 3) three adaptive HHC controllers that automatically update the transfer matrix. A primary conclusion of this series of tests was that the fixed-gain controller was highly successful in reducing vibrations throughout most of the level flight envelope. Shaw et al. ${ }^{5}$ also found that measurements of the sensitivity matrix were highly repeatable. Some of Shaw's success might have been because vibration sensors were placed in the rotating frame.

Despite this demonstrated success with the simple fixed-gain controller, other studies have observed unstable behavior with invariant HHC. ${ }^{6}$ In wind-tunnel tests, gain-scheduled and fixedgain controllers were shown to saturate the control inputs without achieving any vibration reduction. However, several adaptive variants performed very well. ${ }^{7}$ This test also found that open-loop determinations of the sensitivity matrix were not very repeatable and did not necessarily compare with the sensitivity matrix found by adaptive identification. ${ }^{7}$ In a later simulation study, ${ }^{8}$ Molusis considered the effect of nonlinearity on HHC. He attributes the earlier issues with convergence ${ }^{6,7}$ to nonlinearity in the system. Multiple minimum solutions were found in several cases, often causing the controller to converge to a false minimum. A nonlinear relationship between HHC input and vibration output was also considered by means of Volterra series.

The aforementioned wind-tunnel tests were followed by flight tests. ${ }^{9}$ An OH-6A helicopter with an HHC system was flown from zero airspeed to $100 \mathrm{kn}$, using HHC in both open-loop and closedloop modes. These tests showed a significant reduction in helicopter vibrations. However, in transient flight unsatisfactory results were observed. ${ }^{10}$ A HHC system was also flight tested onboard an SA 349 Gazelle. ${ }^{11}$ Both adaptive and fixed-gain controllers were found to be quite successful in reducing vibration during flight, and the adaptive technique had success in maneuvering flight. The successful HHC wind-tunnel and flight tests were all performed with articulated rotors. A subsequent numerical study found that to implement vibration reduction on a hingeless rotor, significantly higher $\mathrm{HHC}$ angles were required, leading to increased blade root moments and pitch link loads. ${ }^{12}$

In an attempt to resolve the apparent discrepancy between the wind-tunnel tests of Shaw and coworkers, ${ }^{3-5}$ Hammond $^{6}$ and Molusis et al., ${ }^{7}$ Nygren and Schrage ${ }^{13}$ conducted a series of numerical simulations with a helicopter aeroelastic response code. This study suggested that fixed-gain control could achieve satisfactory results if the helicopter airspeed were within about $20 \mathrm{kn}$ of the speed at which identification was performed. Constant-thrust maneuvering flight was also studied, where results showed a distinct advantage for adaptive control. The adaptive controller was also found to quickly recover from improper initialization.

Jacklin ${ }^{14}$ has performed an extensive investigation of five system identification algorithms for HHC. The primary focus of the study was to compare several least-squares and Kalman-filter techniques. It was found that generally comparable results were obtained; however, the Kalman-filter methods required a substantial amount of parameter tuning, whereas their least-squares counterpart required much less. The study also found that open-loop identification was the most reliable and could obtain the best vibration reduction results. The controller could achieve minimum vibration even before the sensitivity matrix converged to the correct value. Accurate closedloop identification was found to be more difficult than open-loop identification because the control commands were chosen to control the helicopter vibrations rather than to provide excitation. As vibrations approached the minimum value, identification was complicated by the low signal-to-noise ratio. Probing signals improved the online identification process but produced unacceptable vibration increases.

Hall and Wereley ${ }^{15}$ have developed a continuous-time internalmodel principle ${ }^{16}$ implementation of HHC, which was shown to be, within first approximation, ${ }^{17}$ identical to the classical $\boldsymbol{T}$-matrix 
HHC discussed earlier. This study dealt only with the nonadaptive (invariant) version of the HHC algorithm. Implementation of this approach required the transfer functions between input and response at different flight conditions - information that can be obtained only experimentally. A similar approach was adopted in Ref. 18 and used for numerical simulations of algorithm stability during vibration reduction.

Sometime after most of this research was performed in the rotorcraft field, an almost identical algorithm was applied in Ref. 19 to the problem of balancing a rotor on magnetic bearings. In this study, the term convergent control was used for the HHC algorithm, in reference to the requirement of achieving a steady-state solution before feeding back information to the controller. Robustness bounds were also explored.

The preceding literature review has highlighted some of the more relevant developments in the history of the HHC algorithm. Recent applied work with this algorithm includes studies of blade-vortexinteraction (BVI) noise and vibration reduction, ${ }^{20}$ dynamic stall, ${ }^{21}$ a highly nonlinear phenomenon, and also actuator saturation. ${ }^{22}$ Notable among these studies is the scarcity of careful examination of this algorithm from a more rigorous control perspective. The primary reasons for the success of the HHC algorithm are because of its relative simplicity, its effectiveness, and, to some extent, its robustness. For general broadband disturbance rejection, there exist fundamental tradeoffs that imply that disturbance reduction over a frequency range entails amplification at other frequencies. ${ }^{23}$ However, in the case where disturbances have specific, known frequencies (tonal disturbances), very high gain can be applied at these frequencies of disturbance, and excellent reduction can be achieved without significant compromises. Thus, this disturbance rejection algorithm is particularly suitable for rotorcraft because the disturbance is largely tonal, and the frequency of the disturbance is known a priori.

The paper has the following objectives:

1) Discuss three versions of the HHC algorithm: a) the classical, invariant version; b) the adaptive version, wherein a recursive-leastsquares method is used for online identification; and c) a new relaxed variant.

2) Analyze the robustness of all three versions of HHC, including conditions for stability and convergence. Both additive and multiplicative model uncertainty are considered.

3) Illustrate the use of the preceding variants of HHC through simulations of helicopter vibration control with an actively controlled flap, and identify differences between the variants.

In achieving these objectives the paper provides a more comprehensive theoretical foundation, from a control theory perspective, for this successful algorithm while enhancing our understanding of its effectiveness and simplicity.

\section{HHC Algorithm}

The HHC algorithm is based on the assumption that the helicopter can be represented by a linear model relating output $z$ to control inputs $\boldsymbol{u}$. In previous studies dealing with a four-bladed rotor, ${ }^{1,20,21,24}$ the output $z$ consisted of $4 / \mathrm{rev}$ vibration levels, while the input vector $\boldsymbol{u}$ contained harmonic control inputs including $2 / \mathrm{rev}, 3 / \mathrm{rev}, 4 / \mathrm{rev}$, and $5 / \mathrm{rev}$ components. These relations are illustrated schematically in Fig. 2. Note that the vector $z$ of length $2 p$ is made up of the sine and cosine components of the $p$ harmonic elements in the output signal $z(t)$. Similarly, the vector $\boldsymbol{u}$ of length $2 m$ is made up of the sine and cosine components of $m$ harmonic elements in the control input signal $u(t)$.

The stability analyses that follow consider only the stability of the algorithm, not the closed-loop aeromechanical/aeroelastic stability of the rotor system when control is engaged. The aeromechanical stability problem has been examined numerically in Ref. 18.

The HHC algorithm is a frequency-domain approach applied to disturbances (vibrations or noise) having known frequency content that is usually associated with the number of blades and is connected to the blade passage frequency $N_{b} / \mathrm{rev}$. In the HHC algorithm, the measurement of the plant output and update of the control input are not performed continuously, but rather at specific times $t_{k}=k \tau$,

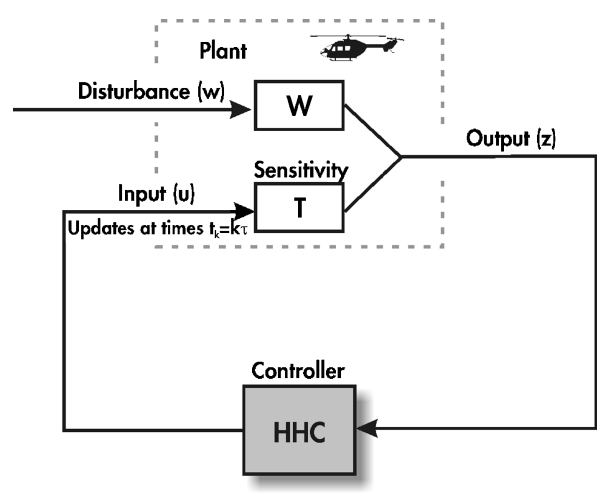

Fig. 3 Higher-harmonic-control architecture.

where $\tau$ is the time interval between updates during which the plant output reaches a steady level. In actual implementation of the algorithm, this time interval can be one or more revolutions. During the application of the algorithm, the system is allowed to reach a steadystate (converged) condition, and measures of the vibratory response amplitude and phase are used to determine the amplitude and phase of the required control input signal for vibration reduction. In actual helicopter flight, maneuvers or other transient behavior could prevent the steady-state condition from being satisfied and compromise the performance of the algorithm.

A diagram of the HHC system as implemented in a helicopter is shown in Fig. 3. With the introduction of a disturbance $\boldsymbol{w}$, representative of the helicopter operating condition, and the observation that system updates are performed at times $t_{k}=k \tau$, the system dynamics are now given by

$$
\boldsymbol{z}_{k}=\boldsymbol{T} \boldsymbol{u}_{k}+\boldsymbol{W} \boldsymbol{w}
$$

In the initial condition, when $k=0$, this can be written as

$$
z_{0}=\boldsymbol{T} u_{0}+W w
$$

Subtracting Eq. (2) from Eq. (1) to eliminate the unknown $w$ yields

$$
\boldsymbol{z}_{k}=\boldsymbol{z}_{0}+\boldsymbol{T}\left(\boldsymbol{u}_{k}-\boldsymbol{u}_{0}\right)
$$

Equation (3) is referred to by Johnson as the global model of helicopter response. ${ }^{2}$ Note that this model depends on the assumption of linearity and that the sensitivity $\boldsymbol{T}$ (and the starting condition $\boldsymbol{z}_{0}$ ) are invariant and known without error either through an identification procedure or direct measurement (offline identification).

The sensitivity $\boldsymbol{T}$ is given by

$$
\boldsymbol{T}=\frac{\partial z}{\partial \boldsymbol{u}}
$$

The controller is based on a general quadratic cost function

$$
J\left(z_{k}, \boldsymbol{u}_{k}\right)=\boldsymbol{z}_{k}^{T} \boldsymbol{Q} \boldsymbol{z}_{k}+2 \boldsymbol{z}_{k}^{T} \boldsymbol{S} \boldsymbol{u}_{k}+\boldsymbol{u}_{k}^{T} \boldsymbol{R} \boldsymbol{u}_{k}
$$

However, in most applications the cross-weighting term in Eq. (5) is neglected, and the cost function simplifies to

$$
J\left(\boldsymbol{z}_{k}, \boldsymbol{u}_{k}\right)=\boldsymbol{z}_{k}^{T} \boldsymbol{Q} \boldsymbol{z}_{k}+\boldsymbol{u}_{k}^{T} \boldsymbol{R} \boldsymbol{u}_{k}
$$

The optimal control law is determined from the requirement

$$
\frac{\partial J\left(\boldsymbol{z}_{k}, \boldsymbol{u}_{k}\right)}{\partial \boldsymbol{u}_{k}}=0
$$

which yields the optimal control law $\boldsymbol{u}_{k, \text { opt }}$, given by

$$
\boldsymbol{u}_{k, \mathrm{opt}}=-\left(\boldsymbol{T}^{T} \boldsymbol{Q T}+\boldsymbol{R}\right)^{-1}\left(\boldsymbol{T}^{T} \boldsymbol{Q}\right)\left(\boldsymbol{z}_{0}-\boldsymbol{T} \boldsymbol{u}_{0}\right)
$$

Combining Eqs. (6) and (8), the minimum cost is found to be

$$
J\left(z_{k}, \boldsymbol{u}_{k, \text { opt }}\right)=\left(z_{0}-\boldsymbol{T} \boldsymbol{u}_{0}\right)^{T}\left[\boldsymbol{Q}-(\boldsymbol{Q T}) \boldsymbol{D}^{-1}\left(\boldsymbol{T}^{T} \boldsymbol{Q}\right)\right]\left(z_{0}-\boldsymbol{T} \boldsymbol{u}_{0}\right)
$$


where

$$
D=T^{T} Q T+R
$$

This is the most basic version of the HHC algorithm, and it yields an explicit relation for optimal control input.

It is also useful to consider another, recursive, form of Eq. (3), where subsequent control updates are written as

$$
\boldsymbol{z}_{k+1}=\boldsymbol{z}_{k}+\boldsymbol{T}\left(\boldsymbol{u}_{k+1}-\boldsymbol{u}_{k}\right)
$$

In this equation, the index $k$ refers to the controller update number, an integer corresponding to each time the control algorithm gets new values of the input vector $\boldsymbol{z}_{k}$ and output vector $\boldsymbol{u}_{k}$. Note that Eq. (3) is a special case of Eq. (11), where $k=0$. However, from Eq. (8), $\boldsymbol{u}_{k, \text { opt }}$ is independent of $k$ and remains constant for all future control updates $k \geq 1$. Equation (11) is referred to by Johnson ${ }^{2}$ as the local controller, and it represents a linearization of the response about the current control value.

\section{Convergence Analysis}

A recursive form of the optimal control law is developed to demonstrate the one-step convergence property of HHC. Substituting Eq. (8) into Eqs. (3) and (5), the optimal value of $z_{k}$ for all $k \geq 1$ is given by

$$
z_{k, \text { opt }}=\left[\boldsymbol{I}-\boldsymbol{T} \boldsymbol{D}^{-1}\left(\boldsymbol{T}^{T} \boldsymbol{Q}+\boldsymbol{S}^{T}\right)\right]\left(z_{0}-\boldsymbol{T} \boldsymbol{u}_{0}\right)
$$

and thus

$$
\begin{aligned}
& J\left(\boldsymbol{z}_{k, \text { opt }}, \boldsymbol{u}_{k, \mathrm{opt}}\right)=\left(\boldsymbol{z}_{0}-\boldsymbol{T} \boldsymbol{u}_{0}\right)^{T}\left[\boldsymbol{Q}-(\boldsymbol{Q} \boldsymbol{T}+\boldsymbol{S}) \boldsymbol{D}^{-1}\left(\boldsymbol{T}^{T} \boldsymbol{Q}+\boldsymbol{S}^{T}\right)\right] \\
& \times\left(\boldsymbol{z}_{0}-\boldsymbol{T} \boldsymbol{u}_{0}\right)
\end{aligned}
$$

These values are attained after the first update.

Using Eq. (3), the optimal control law of Eq. (8) can be expressed recursively as

$$
\boldsymbol{u}_{k+1, \mathrm{opt}}=-\boldsymbol{D}^{-1}\left(\boldsymbol{T}^{T} \boldsymbol{Q}+\boldsymbol{S}^{T}\right)\left(\boldsymbol{z}_{k, \mathrm{opt}}-\boldsymbol{T} \boldsymbol{u}_{k, \mathrm{opt}}\right)
$$

The state-space representation of the system dynamics with the optimal control law is

$$
\left[\begin{array}{c}
\boldsymbol{z}_{k+1, \mathrm{opt}} \\
\boldsymbol{u}_{k+1, \mathrm{opt}}
\end{array}\right]=\boldsymbol{A}\left[\begin{array}{c}
\boldsymbol{z}_{k, \mathrm{opt}} \\
\boldsymbol{u}_{k, \mathrm{opt}}
\end{array}\right]
$$

where $\boldsymbol{A}$ is a $2(p+m) \times 2(p+m)$ matrix defined by

$$
\boldsymbol{A}=\left[\begin{array}{cc}
\boldsymbol{I}_{2 p}-\boldsymbol{T M} & -\left(\boldsymbol{I}_{2 p}-\boldsymbol{T M}\right) \boldsymbol{T} \\
-\boldsymbol{M} & \boldsymbol{M T}
\end{array}\right]
$$

and $\boldsymbol{M}$ is a $2 m \times 2 p$ matrix defined by

$$
\boldsymbol{M}=\boldsymbol{D}^{-1}\left(\boldsymbol{T}^{T} \boldsymbol{Q}+\boldsymbol{S}^{T}\right)
$$

Note that

$$
\begin{gathered}
\boldsymbol{A}^{2}=\left[\begin{array}{cc}
\left(\boldsymbol{I}_{2 p}-\boldsymbol{T M}\right)^{2}+\left(\boldsymbol{I}_{2 p}-\boldsymbol{T M}\right) \boldsymbol{T M} & -\left(\boldsymbol{I}_{2 p}-\boldsymbol{T M}\right)^{2} \boldsymbol{T}-\left(\boldsymbol{I}_{2 p}-\boldsymbol{T M}\right) \boldsymbol{T M T} \\
-\boldsymbol{M}\left(\boldsymbol{I}_{2 p}-\boldsymbol{T M}\right)-\boldsymbol{M T M} & \boldsymbol{M}\left(\boldsymbol{I}_{2 p}-\boldsymbol{T M}\right) \boldsymbol{T}+(\boldsymbol{M T})^{2}
\end{array}\right] \\
=\left[\begin{array}{cc}
\boldsymbol{I}_{2 p}-\boldsymbol{T M} & -\left(\boldsymbol{I}_{2 p}-\boldsymbol{T M}\right) \boldsymbol{T} \\
-\boldsymbol{M} & \boldsymbol{M} \boldsymbol{T}
\end{array}\right]=\boldsymbol{A}
\end{gathered}
$$

Hence $\boldsymbol{A}$ is an idempotent matrix (a matrix such that $\boldsymbol{A}^{2}=\boldsymbol{A}$ ), and its eigenvalues are either 0 or 1 . To show this, note that $\boldsymbol{A}$ can be decomposed as

$$
\boldsymbol{A}=\left[\begin{array}{cc}
\boldsymbol{I}_{2 p} & \boldsymbol{T} \\
0 & \boldsymbol{I}_{2 m}
\end{array}\right]\left[\begin{array}{cc}
\boldsymbol{I}_{2 p} & 0 \\
-\boldsymbol{M} & 0_{2 m}
\end{array}\right]\left[\begin{array}{cc}
\boldsymbol{I}_{2 p} & -\boldsymbol{T} \\
0 & \boldsymbol{I}_{2 m}
\end{array}\right]
$$

which implies that the spectral radius of $\boldsymbol{A}$

$$
\max _{1 \leq i \leq n}\left|\lambda_{i}\right|=\operatorname{sprad}(\boldsymbol{A})=\rho_{s}(\boldsymbol{A})
$$

where $\boldsymbol{A}$ has $n$ eigenvalues $\lambda_{i}$, can be interpreted as the union of $\rho_{s}\left(\boldsymbol{I}_{2 p}\right)$ and $\rho_{s}\left(\mathbf{0}_{2} m\right)$ :

$$
\rho_{s}(\boldsymbol{A})=\rho_{s}\left(\boldsymbol{I}_{2 p}\right) \cup \rho_{s}\left(\mathbf{0}_{2 m}\right)
$$

Recall that the eigenvalues of the identity matrix are $\lambda_{I_{2} p}=$ $\{1, \ldots, 1\}$, and for the zero matrix $\lambda_{0_{2} m}=\{0, \ldots, 0\}$. Observing the block structure of the three matrices of Eq. (19), all eigenvalues of the first and third matrix are 1, and eigenvalues of the central matrix must be 0 or 1 . With initial conditions $z_{0}$ and $\boldsymbol{u}_{0}$, Eq (15) implies that

$$
\begin{gathered}
{\left[\begin{array}{l}
z_{1, \mathrm{opt}} \\
\boldsymbol{u}_{1, \mathrm{opt}}
\end{array}\right]=\boldsymbol{A}\left[\begin{array}{l}
z_{0} \\
\boldsymbol{u}_{0}
\end{array}\right]} \\
{\left[\begin{array}{l}
z_{2, \mathrm{opt}} \\
\boldsymbol{u}_{2, \mathrm{opt}}
\end{array}\right]=\boldsymbol{A}\left[\begin{array}{l}
z_{1, \mathrm{opt}} \\
\boldsymbol{u}_{1, \mathrm{opt}}
\end{array}\right]=\boldsymbol{A}^{2}\left[\begin{array}{l}
z_{0} \\
\boldsymbol{u}_{0}
\end{array}\right]=\boldsymbol{A}\left[\begin{array}{l}
z_{0} \\
\boldsymbol{u}_{0}
\end{array}\right]=\left[\begin{array}{l}
z_{1, \mathrm{opt}} \\
\boldsymbol{u}_{1, \mathrm{opt}}
\end{array}\right]}
\end{gathered}
$$

Similarly, for all $k=1,2, \ldots$,

$$
\left[\begin{array}{c}
\boldsymbol{z}_{k, \mathrm{opt}} \\
\boldsymbol{u}_{k, \mathrm{opt}}
\end{array}\right]=\boldsymbol{A}\left[\begin{array}{c}
\boldsymbol{z}_{0} \\
\boldsymbol{u}_{0}
\end{array}\right]=\left[\begin{array}{c}
(\boldsymbol{I}-\boldsymbol{T} \boldsymbol{M})\left(\boldsymbol{z}_{0}-\boldsymbol{T} \boldsymbol{u}_{0}\right) \\
-\boldsymbol{M}\left(\boldsymbol{z}_{0}-\boldsymbol{T} \boldsymbol{u}_{0}\right)
\end{array}\right]
$$

This confirms the fact that optimum values $\boldsymbol{u}_{k, \text { opt }}$ in Eq. (8) and $\boldsymbol{z}_{k, \text { opt }}$ in Eq. (12) are attained after the first update.

\section{Robustness of the HHC Algorithm}

The development of the HHC control algorithm assumed that the sensitivity matrix $\boldsymbol{T}$ was known without error. In practical implementation, this is unlikely. Changes in flight condition or nonlinearities can easily cause the system behavior to deviate from this assumption. When $\boldsymbol{T}$ is uncertain, the implementation of HHC with erroneous $\boldsymbol{T}$ can result in degradation of performance and possible instability. If $\boldsymbol{T}$ is not known exactly, and only an estimate $\boldsymbol{T}$ of $\boldsymbol{T}$ is given, the control law defined by Eq. (14) becomes

$$
\hat{\boldsymbol{u}}_{k+1}=-\hat{\boldsymbol{M}}\left(\boldsymbol{z}_{k}-\hat{\boldsymbol{T}} \hat{\boldsymbol{u}}_{k}\right)
$$

where

$$
\hat{\boldsymbol{M}}=\hat{\boldsymbol{D}}^{-1}\left(\hat{\boldsymbol{T}}^{T} \boldsymbol{Q}+\boldsymbol{S}^{T}\right)
$$

To develop a condition for the stability of the algorithm, a statespace representation of the system dynamics must be introduced, from Eq. (25):

$$
\left[\begin{array}{l}
\boldsymbol{z}_{k+1} \\
\hat{\boldsymbol{u}}_{k+1}
\end{array}\right]=\hat{\boldsymbol{A}}\left[\begin{array}{l}
\boldsymbol{z}_{k} \\
\hat{\boldsymbol{u}}_{k}
\end{array}\right]
$$

where $\hat{A}$ is defined by

$$
\hat{\boldsymbol{A}}=\left[\begin{array}{cc}
\left(\boldsymbol{I}_{2 p}-\boldsymbol{T} \hat{\boldsymbol{M}}\right) & \boldsymbol{T}\left(\hat{\boldsymbol{M}} \hat{\boldsymbol{T}}-\boldsymbol{I}_{2 p}\right) \\
-\hat{\boldsymbol{M}} & \hat{\boldsymbol{M}} \hat{\boldsymbol{T}}
\end{array}\right]
$$

and $\Delta \boldsymbol{T}=\hat{\boldsymbol{T}}-\boldsymbol{T}$. 
It is useful to factor $\hat{\boldsymbol{A}}$ as

$$
\hat{\boldsymbol{A}}=\left[\begin{array}{cc}
\boldsymbol{I}_{2 p} & \boldsymbol{T} \\
0 & \boldsymbol{I}_{2 m}
\end{array}\right]\left[\begin{array}{cc}
\boldsymbol{I}_{2 p} & 0 \\
-\hat{\boldsymbol{M}} & \hat{\boldsymbol{M}} \Delta \boldsymbol{T}
\end{array}\right]\left[\begin{array}{cc}
\boldsymbol{I}_{2 p} & -\boldsymbol{T} \\
0 & \boldsymbol{I}_{2 m}
\end{array}\right]
$$

which shows that

$$
\max _{1 \leq i \leq n}\left|\left(\lambda_{\hat{A}}\right)_{i}\right|=\rho_{s}(\hat{\boldsymbol{A}})=\rho_{s}\left(\boldsymbol{I}_{2 p}\right) \cup \rho_{s}(\hat{\boldsymbol{M}} \Delta \boldsymbol{T})
$$

Equation (30) yields insight on the stability of the system. Note that stability of the algorithm requires that the eigenvalues of $\hat{\boldsymbol{M}} \Delta \boldsymbol{T}$ be such that

$$
\max _{1 \leq i \leq n}\left|\left(\lambda_{\hat{M} \Delta T}\right)_{i}\right|=\rho_{s}(\hat{\boldsymbol{M}} \Delta \boldsymbol{T})<1
$$

Using Eq. (6), the spectral radius $\rho_{s}(\hat{\boldsymbol{M}} \Delta \boldsymbol{T})$ can be evaluated, if an operator $\sigma_{\max }$ is defined as the largest singular value of the matrix $\boldsymbol{A}$

$$
\sigma_{\max }(\boldsymbol{A})=\max _{1 \leq i \leq n}\left[\sqrt{\left(\lambda_{A^{\star} A}\right)_{i}}\right]
$$

where $\boldsymbol{A}^{\star}$ is the adjoint or conjugate-transpose matrix of $\boldsymbol{A}$, $\boldsymbol{A}^{\star}=(\overline{\boldsymbol{A}})^{T}$. Similarly, an operator $\sigma_{\min }$ can be defined as the smallest singular value of matrix. By using these operators to expand the stability condition,

$$
\begin{aligned}
\rho_{s}(\hat{\boldsymbol{M}} \Delta \boldsymbol{T}) & =\rho_{s}\left[\left(\hat{\boldsymbol{T}}^{T} \boldsymbol{Q} \hat{\boldsymbol{T}}+\boldsymbol{R}\right)^{-1} \hat{\boldsymbol{T}}^{T} \boldsymbol{Q} \Delta \boldsymbol{T}\right] \\
& \leq \sigma_{\max }\left[\left(\hat{\boldsymbol{T}}^{T} \boldsymbol{Q} \hat{\boldsymbol{T}}+\boldsymbol{R}\right)^{-1} \hat{\boldsymbol{T}}^{T} \boldsymbol{Q} \Delta \boldsymbol{T}\right] \\
& \leq \frac{\sigma_{\max }(\hat{\boldsymbol{T}}) \sigma_{\max }(\boldsymbol{Q}) \sigma_{\max }(\Delta \boldsymbol{T})}{\sigma_{\min }\left(\hat{\boldsymbol{T}}^{T} \boldsymbol{Q} \hat{\boldsymbol{T}}+\boldsymbol{R}\right)} \\
& \leq \frac{\left[\sigma_{\max }(\boldsymbol{T})+\sigma_{\max }(\Delta \boldsymbol{T})\right] \sigma_{\max }(\boldsymbol{Q}) \sigma_{\max }(\Delta \boldsymbol{T})}{\sigma_{\min }(\boldsymbol{R})}
\end{aligned}
$$

and it can be shown that, if

$$
\sigma_{\max }(\Delta \boldsymbol{T})<-\sigma_{\max }(\boldsymbol{T}) / 2+\frac{1}{2} \sqrt{\sigma_{\max }(\boldsymbol{T})^{2}+4\left[\sigma_{\min }(R) / \sigma_{\max }(Q)\right]}
$$

then sprad $(\hat{\boldsymbol{M}} \Delta \boldsymbol{T})<1$. The matrix $\boldsymbol{Q}$ consists of weights on the parameters to be reduced (such as vibration or noise levels) while the matrix $\boldsymbol{R}$ consists of weights on the control inputs (such as flap deflections). If $\sigma_{\min }(\boldsymbol{R}) / \sigma_{\max }(\boldsymbol{Q})$ is large (minimum energy control), then according to Eq. (34) HHC control possesses a high degree of robustness, and a sufficient condition for the stability of HHC algorithm is approximately given by

$$
\sigma_{\max }(\Delta \boldsymbol{T})<\sqrt{\sigma_{\min }(\boldsymbol{R}) / \sigma_{\max }(\boldsymbol{Q})}
$$

However, if $\sigma_{\min }(\boldsymbol{R}) / \sigma_{\max }(\boldsymbol{Q})$ is small (cheap control), then Eq. (34) implies that robustness is somewhat compromised.

The explicit stability condition of Eq. (35) lends insight into the behavior of the algorithm. In a later section of this paper, it will be shown that the algorithm can be made stable even in situations where $\boldsymbol{T}$ varies significantly.

The preceding analysis has shown that the stability of the algorithm depends on the degree to which $\boldsymbol{T}$ is correctly estimated or the choice of control weightings $\boldsymbol{Q}$ and $\boldsymbol{R}$. The behavior of the objective function $J$ has not yet been considered. That is, even if the algorithm is stable, having only an estimate of $\boldsymbol{T}$ can affect the attainable minimum. Thus, a convergence analysis is required. Consider Eq. (29), which can be extended to show

$$
\hat{\boldsymbol{A}}^{k}=\left[\begin{array}{cc}
\boldsymbol{I}_{2 p} & \boldsymbol{T} \\
\mathbf{0} & \boldsymbol{I}_{2 m}
\end{array}\right]\left[\begin{array}{cc}
\boldsymbol{I} & 0 \\
-\sum_{i=1}^{k-1}(\hat{\boldsymbol{M}} \Delta \boldsymbol{T})^{i} \hat{\boldsymbol{M}} & (\hat{\boldsymbol{M}} \Delta \boldsymbol{T})^{k}
\end{array}\right]\left[\begin{array}{cc}
\boldsymbol{I}_{2 p} & -\boldsymbol{T} \\
0 & \boldsymbol{I}_{2 m}
\end{array}\right]
$$

Now it is assumed that HHC is stable, that is, Eq. (31) is satisfied. In this case,

$$
\lim _{k \rightarrow \infty}(\hat{\boldsymbol{M}} \Delta \boldsymbol{T})^{k}=0
$$

and one can define a matrix $\boldsymbol{\Gamma}_{s}$ such that

$$
\boldsymbol{\Gamma}_{s}=\lim _{k \rightarrow \infty} \sum_{i=1}^{k-1}(\hat{\boldsymbol{M}} \Delta \boldsymbol{T})^{i}=(\boldsymbol{I}-\hat{\boldsymbol{M}} \Delta \boldsymbol{T})^{-1}
$$

Hence Eqs. (36-38) imply that

$$
\lim _{k \rightarrow \infty} \hat{\boldsymbol{A}}^{k}=\left[\begin{array}{cc}
\boldsymbol{I}-\boldsymbol{T} \boldsymbol{\Gamma}_{s} \hat{\boldsymbol{M}} & \boldsymbol{T}\left(\boldsymbol{\Gamma}_{s} \hat{\boldsymbol{M}} \boldsymbol{T}-\boldsymbol{I}\right) \\
-\boldsymbol{\Gamma}_{s} \hat{\boldsymbol{M}} & \boldsymbol{\Gamma}_{s} \hat{\boldsymbol{M}} \boldsymbol{T}
\end{array}\right]
$$

The limiting values of $\boldsymbol{z}_{k}$ and $\hat{\boldsymbol{u}}_{k}$ are given by

$$
\lim _{k \rightarrow \infty}\left[\begin{array}{l}
\boldsymbol{z}_{k} \\
\hat{\boldsymbol{u}}_{k}
\end{array}\right]=\lim _{k \rightarrow \infty} \hat{\boldsymbol{A}}^{k}\left[\begin{array}{c}
\boldsymbol{z}_{0} \\
\hat{\boldsymbol{u}}_{0}
\end{array}\right]=\left[\begin{array}{c}
\left(\boldsymbol{I}-\boldsymbol{T} \boldsymbol{\Gamma}_{s} \hat{\boldsymbol{M}}\right)\left(z_{0}-\boldsymbol{T} \hat{\boldsymbol{u}}_{0}\right) \\
-\boldsymbol{\Gamma}_{s} \hat{\boldsymbol{M}}\left(\boldsymbol{z}_{0}-\boldsymbol{T} \hat{\boldsymbol{u}}_{0}\right)
\end{array}\right]
$$

Next, define the limiting cost $J_{\hat{T}, \infty}$ by

$$
J_{\hat{T}, \infty}=\lim _{k \rightarrow \infty} J\left(\boldsymbol{z}_{k}, \hat{\boldsymbol{u}}_{k}\right)
$$

Substituting Eq. (40) into Eq. (5) yields

$$
\begin{aligned}
J_{\hat{T}, \infty} & =\left(z_{0}-\boldsymbol{T} \hat{\boldsymbol{u}}_{0}\right)^{T}\left[\boldsymbol{Q}-\boldsymbol{Q T} \boldsymbol{\Gamma}_{s} \hat{\boldsymbol{M}}-\left(\boldsymbol{Q} \boldsymbol{T} \boldsymbol{\Gamma}_{s} \hat{\boldsymbol{M}}\right)^{T}+\hat{\boldsymbol{M}}^{T} \boldsymbol{\Gamma}_{s}^{T} \boldsymbol{D} \boldsymbol{\Gamma}_{s} \hat{\boldsymbol{M}}\right] \\
& \times\left(z_{0}-\boldsymbol{T} \hat{\boldsymbol{u}}_{0}\right)
\end{aligned}
$$

Note that

$$
\left(\boldsymbol{D}^{-\frac{1}{2}} \boldsymbol{T}^{T} \boldsymbol{Q}-\boldsymbol{D}^{\frac{1}{2}} \boldsymbol{\Gamma}_{s} \hat{\boldsymbol{M}}\right)^{T}\left(\boldsymbol{D}^{-\frac{1}{2}} \boldsymbol{T}^{T} \boldsymbol{Q}-\boldsymbol{D}^{\frac{1}{2}} \boldsymbol{\Gamma}_{s} \hat{\boldsymbol{M}}\right) \geq 0
$$

and hence

$$
\boldsymbol{Q T D ^ { - 1 }} \boldsymbol{T}^{T} \boldsymbol{Q} \geq \boldsymbol{Q T} \boldsymbol{\Gamma}_{s} \hat{\boldsymbol{M}}+\left(\boldsymbol{Q T T} \boldsymbol{\Gamma}_{s} \hat{\boldsymbol{M}}\right)^{T}-\hat{\boldsymbol{M}}^{T} \boldsymbol{\Gamma}_{s}^{T} \boldsymbol{D} \boldsymbol{\Gamma}_{s} \hat{\boldsymbol{M}}
$$

Assuming $\hat{\boldsymbol{u}}_{0}=\boldsymbol{u}_{0}$, Eqs. (9), (42), and (44) yield

$$
J_{\hat{T}, \infty} \geq J\left(z, \boldsymbol{u}_{k, \mathrm{opt}}\right)
$$

which confirms the expected fact that the performance is not better than the optimal performance obtained when $\boldsymbol{T}$ is exactly known.

Now, consider the case where the estimated value $\hat{\boldsymbol{T}}$ of $\boldsymbol{T}$ involves a multiplicative error, that is,

$$
\hat{\boldsymbol{T}}=\boldsymbol{T}\left(\boldsymbol{I}+\Delta \boldsymbol{T}_{\mathrm{mul}}\right)
$$

where $\Delta \boldsymbol{T}$ is a $2 m \times 2 m$ matrix. Then $\hat{\boldsymbol{T}}$ can be expressed equivalently by $\hat{\boldsymbol{T}}=\boldsymbol{T}+\Delta \boldsymbol{T}$, where

$$
\Delta \boldsymbol{T}=\boldsymbol{T} \Delta \boldsymbol{T}_{\mathrm{mul}}
$$

A sufficient condition for stability of HHC is

$$
\rho_{s}\left(\hat{\boldsymbol{M}} \boldsymbol{T} \Delta \boldsymbol{T}_{\mathrm{mul}}\right)<1
$$

Substituting Eq. (47) into Eq. (33) yields

$$
\begin{aligned}
\rho_{s}\left(\hat{\boldsymbol{M}} \boldsymbol{T} \Delta \boldsymbol{T}_{\mathrm{mul}}\right) & =\rho_{s}\left[\left(\hat{\boldsymbol{T}}^{T} \boldsymbol{Q} \hat{\boldsymbol{T}}+\boldsymbol{R}\right)^{-1} \hat{\boldsymbol{T}}^{T} \boldsymbol{Q} \boldsymbol{T} \Delta \boldsymbol{T}_{\mathrm{mul}}\right] \\
& \leq \frac{\sigma_{\max }^{2}(\boldsymbol{T}) \sigma_{\max }(\boldsymbol{Q}) \sigma_{\max }\left(\Delta \boldsymbol{T}_{\mathrm{mul}}\right)\left[\sigma_{\max }\left(\Delta \boldsymbol{T}_{\mathrm{mul}}\right)+1\right]}{\sigma_{\min }(\boldsymbol{R})}
\end{aligned}
$$

Finally, it can be shown that the HHC algorithm is stable if

$$
\sigma_{\max }\left(\Delta \boldsymbol{T}_{\mathrm{mul}}\right)<-\frac{1}{2}+\frac{1}{2} \sqrt{1+\frac{4 \sigma_{\min }(\boldsymbol{R})}{\sigma_{\max }^{2}(\boldsymbol{T}) \sigma_{\max }(\boldsymbol{Q})}}
$$




\section{Relaxed HHC for Enhanced Robustness}

A promising alternative to conventional $\mathrm{HHC}$ is designated "relaxed HHC," where a relaxation coefficient is introduced into the algorithm. This technique was pioneered by Depailler ${ }^{21}$ and mentioned in a computer program user's manual but never fully developed. This variation on the HHC algorithm has proved itself useful under a highly nonlinear condition induced by dynamic stall, where any estimate of $\boldsymbol{T}$ is uncertain. This variant of the algorithm compromises the one-step convergence property of the algorithm shown earlier, but improves robustness. Recall that when $\boldsymbol{T}$ is known the optimal control law $\boldsymbol{u}_{k+1}$ from Eq. (14) can be expressed as

$$
\boldsymbol{u}_{k+1}=\boldsymbol{u}_{k}+\Delta \boldsymbol{u}_{k}
$$

where one can define

$$
\Delta \boldsymbol{u}_{k}=-\boldsymbol{M} \boldsymbol{z}_{k}+(\boldsymbol{M T}-\boldsymbol{I}) \boldsymbol{u}_{k}
$$

Replacing $\Delta \boldsymbol{u}_{k}$ in Eq. (51) by $\alpha \Delta \boldsymbol{u}_{k, \alpha}$ yields the relaxed control update law

$$
\boldsymbol{u}_{k+1, \alpha}=\boldsymbol{u}_{k, \alpha}+\alpha \Delta \boldsymbol{u}_{k, \alpha}
$$

where $\boldsymbol{u}_{0, \alpha}=\boldsymbol{u}_{0}$. In this case, the state-space representation is given by

$$
\left[\begin{array}{c}
\boldsymbol{z}_{k+1} \\
\boldsymbol{u}_{k+1, \alpha}
\end{array}\right]=\boldsymbol{A}_{\alpha}\left[\begin{array}{c}
\boldsymbol{z}_{k} \\
\boldsymbol{u}_{k, \alpha}
\end{array}\right]
$$

where the following definition is used:

$$
\boldsymbol{A}_{\alpha}=\left[\begin{array}{cc}
\boldsymbol{I}-\alpha \boldsymbol{T M} & -(\boldsymbol{I}-\alpha \boldsymbol{T M}) \boldsymbol{T}+(1-\alpha) \boldsymbol{T} \\
-\alpha \boldsymbol{M} & \alpha \boldsymbol{M T}+(1-\alpha) \boldsymbol{I}
\end{array}\right]
$$

Note that for $\alpha=1, \boldsymbol{A}_{1}=\boldsymbol{A}$. The matrix $\boldsymbol{A}_{\alpha}$ can be factored as

$$
\boldsymbol{A}_{\alpha}=\left[\begin{array}{cc}
\boldsymbol{I}_{2 p} & \boldsymbol{T} \\
0 & \boldsymbol{I}_{2 m}
\end{array}\right]\left[\begin{array}{cc}
\boldsymbol{I}_{2 p} & 0 \\
-\alpha \boldsymbol{M} & (1-\alpha) \boldsymbol{I}_{2 m}
\end{array}\right]\left[\begin{array}{cc}
\boldsymbol{I}_{2 p} & -\boldsymbol{T} \\
0 & \boldsymbol{I}_{2 m}
\end{array}\right]
$$

which implies

$$
\max _{1 \leq i \leq n}\left|\left(\boldsymbol{\lambda}_{A_{\alpha}}\right)_{i}\right|=\rho_{s}\left(\boldsymbol{A}_{\alpha}\right)=\rho_{s}\left(\boldsymbol{I}_{2 p}\right) \cup \rho_{s}\left[(1-\alpha) \boldsymbol{I}_{2 m}\right]
$$

Note that

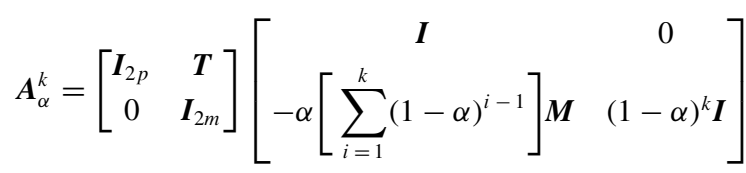

$$
\begin{aligned}
& \times\left[\begin{array}{cc}
\boldsymbol{I}_{2 p} & -\boldsymbol{T} \\
0 & \boldsymbol{I}_{2 m}
\end{array}\right]
\end{aligned}
$$

To analyze Eq. (58), assume that $0<\alpha<2$. In most practical cases, $\alpha<1$, as the step size is reduced by multiplying by the factor $\alpha$. This assumption allows the evaluation of the limits

$$
\begin{aligned}
\lim _{k \rightarrow \infty} \alpha\left[\sum_{i=1}^{k}(1-\alpha)^{i-1}\right] & =1 \\
\lim _{k \rightarrow \infty}(1-\alpha)^{k} & =0
\end{aligned}
$$

hence it follows from Eqs. (58-60) that

$$
\lim _{k \rightarrow \infty} \boldsymbol{A}_{\alpha}^{k}=\boldsymbol{A}
$$

and the steady-state values of $\boldsymbol{z}_{k}$ and $\boldsymbol{u}_{k, \alpha}$ are given by

$$
\lim _{k \rightarrow \infty}\left[\begin{array}{c}
\boldsymbol{z}_{k} \\
\boldsymbol{u}_{k, \alpha}
\end{array}\right]=\lim _{k \rightarrow \infty} \boldsymbol{A}_{\alpha}^{k}\left[\begin{array}{c}
\boldsymbol{z}_{0} \\
\boldsymbol{u}_{0}
\end{array}\right]=\left[\begin{array}{c}
\boldsymbol{z}_{\mathrm{opt}} \\
\boldsymbol{u}_{\mathrm{opt}}
\end{array}\right]
$$

Again, one has to compare the final value of the objective function with its value without the relaxed control. To do so, define the limiting cost $J_{T, \alpha, \infty}$ as

$$
J_{T, \alpha, \infty}=\lim _{k \rightarrow \infty} J\left(z_{k}, \boldsymbol{u}_{k, \alpha}\right)
$$

It thus follows from Eqs. (9) and (62) that

$$
J_{T, \alpha, \infty}=J\left(z, \boldsymbol{u}_{\mathrm{opt}}\right)
$$

Hence, the steady-state values of $\boldsymbol{z}_{k}$ and $\boldsymbol{u}_{k}$ for the relaxed HHC algorithm are the same as the optimal values obtained in a single step using conventional HHC.

Relaxed HHC is only useful in the cases where $\boldsymbol{T}$ is uncertain (it increases convergence time in all other cases), and thus it is useful to perform a robustness analysis in the presence of relaxed control, following the procedure described in the preceding section. When only an estimate $\hat{\boldsymbol{T}}$ of the sensitivity is known, the relaxed HHC update law is given by

$$
\hat{\boldsymbol{u}}_{k+1, \alpha}=\hat{\boldsymbol{u}}_{k, \alpha}+\alpha \Delta \boldsymbol{u}_{k, \alpha}
$$

where $\Delta \boldsymbol{u}_{k, \alpha}$ is defined by

$$
\Delta \boldsymbol{u}_{k}=-\hat{\boldsymbol{M}} \boldsymbol{z}_{k}+(\hat{\boldsymbol{M}} \hat{\boldsymbol{T}}-\boldsymbol{I}) \hat{\boldsymbol{u}}_{k, \alpha}
$$

and $\hat{\boldsymbol{u}}_{0, \alpha}=\boldsymbol{u}_{0}$. The state-space representation of the system dynamics is given by

$$
\left[\begin{array}{c}
\boldsymbol{z}_{k+1} \\
\hat{\boldsymbol{u}}_{k+1, \alpha}
\end{array}\right]=\hat{\boldsymbol{A}}_{\alpha}\left[\begin{array}{c}
\boldsymbol{z}_{k} \\
\hat{\boldsymbol{u}}_{k, \alpha}
\end{array}\right]
$$

where $\hat{\boldsymbol{A}}_{\alpha}$ is defined by

$$
\hat{\boldsymbol{A}}_{\alpha}=\left[\begin{array}{cc}
\boldsymbol{I}-\alpha \boldsymbol{T} \hat{\boldsymbol{M}} & -\alpha \boldsymbol{T}\left(\hat{\boldsymbol{M}} \hat{\boldsymbol{T}}-\boldsymbol{I}_{2 p}\right) \\
-\alpha \hat{\boldsymbol{M}} & \alpha \hat{\boldsymbol{M}} \hat{\boldsymbol{T}}+(1-\alpha) \boldsymbol{I}
\end{array}\right]
$$

The matrix $\hat{\boldsymbol{A}}_{\alpha}$ can be factored as

$$
\hat{\boldsymbol{A}}_{\alpha}=\left[\begin{array}{cc}
\boldsymbol{I}_{2 p} & \boldsymbol{T} \\
0 & \boldsymbol{I}_{2 m}
\end{array}\right]\left[\begin{array}{cc}
\boldsymbol{I}_{2 p} & 0 \\
-\alpha \hat{\boldsymbol{M}} & \alpha \hat{\boldsymbol{M}} \Delta \boldsymbol{T}+(1-\alpha) \boldsymbol{I}_{2 m}
\end{array}\right]\left[\begin{array}{cc}
\boldsymbol{I}_{2 p} & -\boldsymbol{T} \\
0 & \boldsymbol{I}_{2 m}
\end{array}\right]
$$

where $\Delta \boldsymbol{T}=\hat{\boldsymbol{T}}-\boldsymbol{T}$. It follows from Eq. (69) that

$$
\operatorname{spec}\left(\hat{\boldsymbol{A}}_{\alpha}\right)=\operatorname{spec}\left(\boldsymbol{I}_{2 p}\right) \cup \operatorname{spec}\left[\alpha \hat{\boldsymbol{M}} \Delta \boldsymbol{T}+(1-\alpha) \boldsymbol{I}_{2 m}\right]
$$

Hence the relaxed HHC algorithm is stable if and only if

$$
\rho_{s}\left[\alpha \hat{\boldsymbol{M}} \Delta \boldsymbol{T}+(1-\alpha) \boldsymbol{I}_{2 m}\right]<1
$$

Next, consider the eigenvalues of $(\hat{\boldsymbol{M}} \Delta \boldsymbol{M})$,

$$
\operatorname{spec}(\hat{\boldsymbol{M}} \Delta \boldsymbol{T})=\left\{\lambda_{1}, \ldots, \lambda_{2 m}\right\}
$$

such that the eigenvalues of the matrix $\left[\alpha \hat{\boldsymbol{M}} \Delta \boldsymbol{T}+(1-\alpha) \boldsymbol{I}_{2 m}\right]$ can be written as

$$
\operatorname{spec}\left[\alpha \hat{\boldsymbol{M}} \Delta \boldsymbol{T}+(1-\alpha) \boldsymbol{I}_{2 m}\right]=\left\{\lambda_{\alpha_{1}}, \ldots, \lambda_{\alpha_{2 m}}\right\}
$$

where, for all $i=1, \ldots, 2 m$,

$$
\lambda_{\alpha_{i}}=\alpha \lambda_{i}+1-\alpha
$$

Hence Eq. (74) implies that

$$
\left|\lambda_{\alpha_{i}}\right|^{2}=1+\alpha^{2}\left(\sigma_{i}-1\right)^{2}+2 \alpha\left(\sigma_{i}-1\right)+\alpha^{2} \iota_{i}^{2}
$$

where $\sigma_{i}=\operatorname{Re}\left(\lambda_{i}\right)$ and $\iota_{i}=\operatorname{Im}\left(\lambda_{i}\right)$ such that $\lambda_{i}=\sigma_{i}+\iota_{i}$. Next, define $\Lambda_{\min }$ and $\Lambda_{\max }$ as the minimum and maximum real part of the eigenvalues of $\left[\alpha \hat{\boldsymbol{M}} \Delta \boldsymbol{T}+(1-\alpha) \boldsymbol{I}_{2 m}\right]$ by

$$
\begin{aligned}
& \Lambda_{\min }=\min _{i=1, \ldots, 2 m} \sigma_{i} \\
& \Lambda_{\max }=\max _{i=1, \ldots, 2 m} \sigma_{i}
\end{aligned}
$$


Assuming $\Lambda_{\max }<1$, it follows that, for $i=1, \ldots, 2 m$,

$$
\sigma_{i}<1
$$

Hence it follows from Eqs. (75) and (78) that if

$$
0 \leq \alpha<\frac{2\left(1-\sigma_{i}\right)}{\left(1-\sigma_{i}\right)^{2}+\left(\iota_{i}\right)^{2}}
$$

then $\left|\lambda_{\alpha_{i}}\right|<1$. Define

$$
\alpha_{\max }=\min _{i=1, \ldots, 2 m} \frac{2\left(1-\sigma_{i}\right)}{\left(1-\sigma_{i}\right)^{2}+\left(\iota_{i}\right)^{2}}
$$

Hence for all $0 \leq \alpha<\alpha_{\max }$ and $i=1, \ldots, 2 m$, it follows from Eqs. (79) and (80) that

$$
\left|\lambda_{\alpha_{i}}\right|<1
$$

and it follows from Eq. (73) that the condition of Eq. (71) is satisfied.

Alternatively, if $\Lambda_{\min }>1$, then for all $i=1, \ldots, 2 m$,

$$
\sigma_{i}>1
$$

Hence Eqs. (75) and (82) imply that if

$$
\frac{2\left(1-\sigma_{i}\right)}{\left(1-\sigma_{i}\right)^{2}+\left(\iota_{i}\right)^{2}}<\alpha \leq 0
$$

then

$$
\left|\lambda_{\alpha_{i}}\right|<1
$$

Define

$$
\alpha_{\min }=\max _{i=1, \ldots, 2 m} \frac{2\left(1-\sigma_{i}\right)}{\left(1-\sigma_{i}\right)^{2}+\left(\iota_{i}\right)^{2}}
$$

Hence for all $\alpha_{\min }<\alpha \leq 0$, and $i=1, \ldots, 2 m$, it follows from Eqs. (83-85) that

$$
\left|\lambda_{\alpha_{i}}\right|<1
$$

and finally it follows from Eq. (73) that Eq. (71) is once again satisfied. Hence if either $\Lambda_{\max }<1$ or $\Lambda_{\min }>1$, then there exists $\alpha$ such that stability of relaxed HHC control can be guaranteed.

Note that

$$
\begin{aligned}
\hat{\boldsymbol{A}}_{\alpha}^{k} & =\left[\begin{array}{cc}
\boldsymbol{I}_{2 p} & \boldsymbol{T} \\
0 & \boldsymbol{I}_{2 m}
\end{array}\right] \boldsymbol{I} \\
& \times\left[\begin{array}{cc} 
& 0 \\
-\alpha \sum_{i=1}^{k-1}[\alpha \hat{\boldsymbol{M}} \Delta \boldsymbol{T}+(1-\alpha) \boldsymbol{I}]^{i} \hat{\boldsymbol{M}} & {[\alpha \hat{\boldsymbol{M}} \Delta \boldsymbol{T}+(1-\alpha) \boldsymbol{I}]^{k}}
\end{array}\right] \\
& \times\left[\begin{array}{cc}
\boldsymbol{I}_{2 p} & -\boldsymbol{T} \\
0 & \boldsymbol{I}_{2 m}
\end{array}\right]
\end{aligned}
$$

Assuming $\alpha$ is chosen so that Eq. (71) is satisfied, it follows that

$\lim _{k \rightarrow \infty}[\alpha \hat{\boldsymbol{M}} \Delta \boldsymbol{T}+(1-\alpha) \boldsymbol{I}]^{k}=0$

$$
\begin{aligned}
\lim _{k \rightarrow \infty} \sum_{i=1}^{k-1}[\alpha \hat{\boldsymbol{M}} \Delta \boldsymbol{T}+(1-\alpha) \boldsymbol{I}]^{i} \\
\quad=[\boldsymbol{I}-\alpha \hat{\boldsymbol{M}} \Delta \boldsymbol{T}-(1-\alpha) \boldsymbol{I}]^{-1}=\frac{1}{\alpha} \boldsymbol{\Gamma}_{s}
\end{aligned}
$$

where $\boldsymbol{\Gamma}_{s}$ is defined in Eq. (38). Hence from Eqs. (87-89), it follows that

$$
\lim _{k \rightarrow \infty} \hat{\boldsymbol{A}}_{\alpha}^{k}=\left[\begin{array}{cc}
\boldsymbol{I}-\boldsymbol{T} \boldsymbol{\Gamma}_{s} \hat{\boldsymbol{M}} & \boldsymbol{T}\left(\boldsymbol{\Gamma}_{s} \hat{\boldsymbol{M}} \boldsymbol{T}-\boldsymbol{I}\right) \\
-\boldsymbol{\Gamma}_{s} \hat{\boldsymbol{M}} & \boldsymbol{\Gamma}_{s} \hat{\boldsymbol{M}} \boldsymbol{T}
\end{array}\right]
$$

If Eq. (31) is satisfied, then comparing Eqs. (39) and (90) yields

$$
\lim _{k \rightarrow \infty} \hat{\boldsymbol{A}}_{\alpha}^{k}=\lim _{k \rightarrow \infty} \hat{\boldsymbol{A}}^{k}
$$

and hence by using Eq. (40), the steady-state values of $\boldsymbol{z}_{k}$ and $\hat{\boldsymbol{u}}_{k, \alpha}$ are given by

$$
\begin{aligned}
\lim _{k \rightarrow \infty}\left[\begin{array}{c}
\boldsymbol{z}_{k} \\
\hat{\boldsymbol{u}}_{k, \alpha}
\end{array}\right]=\lim _{k \rightarrow \infty} \hat{\boldsymbol{A}}_{\alpha}^{k}\left[\begin{array}{l}
\boldsymbol{z}_{0} \\
\boldsymbol{u}_{0}
\end{array}\right]=\lim _{k \rightarrow \infty} \hat{\boldsymbol{A}}^{k}\left[\begin{array}{l}
\boldsymbol{z}_{0} \\
\boldsymbol{u}_{0}
\end{array}\right] \\
=\left[\begin{array}{c}
\left(\boldsymbol{I}-\boldsymbol{T} \boldsymbol{\Gamma}_{s} \hat{\boldsymbol{M}}\right)\left(\boldsymbol{z}_{0}-\boldsymbol{T} \boldsymbol{u}_{0}\right) \\
-\boldsymbol{\Gamma}_{s} \hat{\boldsymbol{M}}\left(\boldsymbol{z}_{0}-\boldsymbol{T} \boldsymbol{u}_{0}\right)
\end{array}\right]
\end{aligned}
$$

Define the limiting $\cos t J_{\hat{T}, \alpha, \infty}$ by

$$
J_{\hat{T}, \alpha, \infty}=\lim _{k \rightarrow \infty} J\left(\boldsymbol{z}_{k}, \hat{\boldsymbol{u}}_{k, \alpha}\right)
$$

Assuming Eq. (31) is satisfied implies that $J_{\hat{T}, \infty}$ exists, and it follows from Eqs. (45) and (92) that

$$
J_{\hat{T}, \alpha, \infty}=J_{\hat{T}, \infty} \geq J\left(\boldsymbol{z}, \boldsymbol{u}_{\text {opt }}\right)
$$

Hence if $\Delta \boldsymbol{T}$ satisfies the condition of Eq. (31) and that of Eq. (71) for an appropriate $\alpha$, then the limiting value of the objective function obtained using $\mathrm{HHC}$ control and relaxed $\mathrm{HHC}$ control are the same.

\section{Adaptive Control}

Another version of the HHC algorithm, discussed in Ref. 2, is also available. It is known as adaptive or recursive HHC; in this version, the sensitivity $\boldsymbol{T}$ is identified online and is used to implement an adaptive extension of HHC. To pursue this, relative parameters are defined, $\Delta \boldsymbol{z}_{k}$ with length $2 p$ and $\Delta \boldsymbol{u}_{k}$ with length $2 m$, by

$$
\Delta \boldsymbol{z}_{k}=\boldsymbol{z}_{k}-\boldsymbol{z}_{k-1}, \quad \Delta \boldsymbol{u}_{k}=\boldsymbol{u}_{k}-\boldsymbol{u}_{k-1}
$$

and $\Delta \boldsymbol{Z}_{k}$ of size $2 p \times k$ and $\Delta \boldsymbol{U}_{k}$ of size $2 m \times k$ by

$$
\Delta \boldsymbol{Z}_{k}=\left[\begin{array}{lll}
\Delta \boldsymbol{z}_{1} & \cdots & \Delta \boldsymbol{z}_{k}
\end{array}\right], \quad \Delta \boldsymbol{U}_{k}=\left[\begin{array}{lll}
\Delta \boldsymbol{u}_{1} & \cdots & \Delta \boldsymbol{u}_{k}
\end{array}\right]
$$

The relation between the successive updates of vibration levels $z_{k}$ is

$$
\boldsymbol{z}_{k+1}=\boldsymbol{z}_{k}+\boldsymbol{T}\left(\boldsymbol{u}_{k+1}-\boldsymbol{u}_{k}\right)
$$

This can be represented in another form:

$$
\Delta \boldsymbol{z}_{k}=\boldsymbol{T} \Delta \boldsymbol{u}_{k}
$$

Hence, it follows from Eqs. (95) and (98) that

$$
\Delta \boldsymbol{Z}_{k}=\boldsymbol{T} \Delta \boldsymbol{U}_{k}
$$

Assuming $\Delta \boldsymbol{U}_{k} \Delta \boldsymbol{U}_{k}^{T}$ is nonsingular, one can define

$$
\boldsymbol{P}_{k}=\left(\Delta \boldsymbol{U}_{k} \Delta \boldsymbol{U}_{k}^{T}\right)^{-1}
$$

and from Eq. (99) the least-squares estimate $\hat{\boldsymbol{T}}_{\mathrm{LS}_{k}}$ of $\boldsymbol{T}$ is given by

$$
\hat{\boldsymbol{T}}_{\mathrm{LS}_{k}}=\Delta \boldsymbol{Z}_{k} \Delta \boldsymbol{U}_{k}^{T} \boldsymbol{P}_{k}
$$

The recursive least-squares method is used to iteratively update $\hat{\boldsymbol{T}}_{\mathrm{LS}}$ based on the past and current values of $\Delta \boldsymbol{z}_{k}$ and $\Delta \boldsymbol{u}_{k}$. From Eq. (100), it follows that

$$
\begin{aligned}
\boldsymbol{P}_{k+1}^{-1} & =\Delta \boldsymbol{U}_{k+1} \Delta \boldsymbol{U}_{k+1}^{T}=\sum_{i=1}^{k+1} \Delta \boldsymbol{u}_{i} \Delta \boldsymbol{u}_{i}^{T} \\
& =\sum_{i=1}^{k} \Delta \boldsymbol{u}_{i} \Delta \boldsymbol{u}_{i}^{T}+\Delta \boldsymbol{u}_{k+1} \Delta \boldsymbol{u}_{k+1}^{T} \\
& =\boldsymbol{P}_{k}^{-1}+\Delta \boldsymbol{u}_{k+1} \Delta \boldsymbol{u}_{k+1}^{T}
\end{aligned}
$$


rewriting Eq. (101) as

$$
\hat{\boldsymbol{T}}_{\mathrm{LS}_{k}}=\left(\sum_{i=1}^{k} \Delta \boldsymbol{z}_{i} \Delta \boldsymbol{u}_{i}^{T}\right) \boldsymbol{P}_{k}
$$

and replacing $k$ by $k+1$ in Eq. (103) yields

$$
\begin{aligned}
\hat{\boldsymbol{T}}_{\mathrm{LS}_{k+1}} & =\left(\sum_{i=1}^{k+1} \Delta \boldsymbol{z}_{i} \Delta \boldsymbol{u}_{i}^{T}\right) \boldsymbol{P}_{k+1} \\
& =\left(\sum_{i=1}^{k} \Delta \boldsymbol{z}_{i} \Delta \boldsymbol{u}_{i}^{T}+\Delta \boldsymbol{z}_{k+1} \Delta \boldsymbol{u}_{k+1}^{T}\right) \boldsymbol{P}_{k+1}
\end{aligned}
$$

Hence substituting $\boldsymbol{P}_{k}^{-1}$ from Eq. (102) into Eq. (103) yields

$$
\sum_{i=1}^{k} \Delta \boldsymbol{z}_{i} \Delta \boldsymbol{u}_{i}^{T}=\hat{\boldsymbol{T}}_{\mathrm{LS}_{k}}\left(\boldsymbol{P}_{k+1}^{-1}-\Delta \boldsymbol{u}_{k+1} \Delta \boldsymbol{u}_{k+1}^{T}\right)
$$

Substituting Eq. (105) into Eq. (104) yields

$$
\begin{aligned}
\hat{\boldsymbol{T}}_{\mathrm{LS}_{k+1}} & =\left[\hat{\boldsymbol{T}}_{\mathrm{LS}_{k}}\left(\boldsymbol{P}_{k+1}^{-1}-\Delta \boldsymbol{u}_{k+1} \Delta \boldsymbol{u}_{k+1}^{T}\right)+\Delta \boldsymbol{z}_{k+1} \Delta \boldsymbol{u}_{k+1}^{T}\right] \boldsymbol{P}_{k+1} \\
& =\hat{\boldsymbol{T}}_{\mathrm{LS}_{k}}+\left[\Delta \boldsymbol{z}_{k+1}-\hat{\boldsymbol{T}}_{\mathrm{LS}_{k}} \Delta \boldsymbol{u}_{k+1}\right] \Delta \boldsymbol{u}_{k+1}^{T} \boldsymbol{P}_{k+1} \\
& =\hat{\boldsymbol{T}}_{\mathrm{LS}_{k}}+\varepsilon_{k+1} \boldsymbol{K}_{k+1}
\end{aligned}
$$

where

$$
\begin{gathered}
\varepsilon_{k+1}=\Delta \boldsymbol{z}_{k+1}-\hat{\boldsymbol{T}}_{\mathrm{LS}_{k}} \Delta \boldsymbol{u}_{k+1} \\
\boldsymbol{K}_{k+1}=\Delta \boldsymbol{u}_{k+1}^{T} \boldsymbol{P}_{k+1}
\end{gathered}
$$

By using the matrix inversion lemma in Eq. (102) (Ref. 25), $\boldsymbol{P}_{k+1}$ can be expressed recursively as

$$
\boldsymbol{P}_{k+1}=\boldsymbol{P}_{k}-\boldsymbol{P}_{k} \Delta \boldsymbol{u}_{k+1}\left(1+\Delta \boldsymbol{u}_{k+1}^{T} \boldsymbol{P}_{k} \Delta \boldsymbol{u}_{k+1}\right)^{-1} \Delta \boldsymbol{u}_{k+1}^{T} \boldsymbol{P}_{k}
$$

Combining Eqs. (110) and (111) yields

$$
\boldsymbol{K}_{k+1}=\Delta \boldsymbol{u}_{k+1}^{T} \boldsymbol{P}_{k}\left(1+\Delta \boldsymbol{u}_{k+1}^{T} \boldsymbol{P}_{k} \Delta \boldsymbol{u}_{k+1}\right)^{-1}
$$

Because $\boldsymbol{u}_{k} \boldsymbol{u}_{k}^{T}$ is positive semidefinite for all $k$, Eq. (102) implies that if $\Delta \boldsymbol{U}_{k_{0}} \Delta \boldsymbol{U}_{k_{0}}^{T}$ is nonsingular, then $\Delta \boldsymbol{u}_{k} \Delta \boldsymbol{U}_{k}^{T}$ is nonsingular for all $k>k_{0}$. Hence the recursive procedure to determine $\boldsymbol{T}_{\mathrm{LS}_{k}}$ for all $k>k_{0}$ is given by

$$
\begin{gathered}
\boldsymbol{K}_{k+1}=\left(1+\Delta \boldsymbol{u}_{k+1}^{T} P_{k} \Delta \boldsymbol{u}_{k+1}\right)^{-1} \Delta \boldsymbol{u}_{k+1}^{T} P_{k} \\
\hat{\boldsymbol{T}}_{\mathrm{LS}}=\hat{\boldsymbol{T}}_{\mathrm{LS}}+\varepsilon_{k+1} \boldsymbol{K}_{k+1} \\
\boldsymbol{P}_{k+1}=\boldsymbol{P}_{k}\left(\boldsymbol{I}-\Delta \boldsymbol{u}_{k+1} \boldsymbol{K}_{k+1}\right)
\end{gathered}
$$

Note that

$$
\operatorname{rank}\left(\Delta \boldsymbol{U}_{k} \Delta \boldsymbol{U}_{k}^{T}\right)=\operatorname{rank}\left(\Delta \boldsymbol{U}_{k}\right) \leq \min (k, 2 m)
$$

Because $\Delta \boldsymbol{U}_{k} \Delta \boldsymbol{U}_{k}^{T}$ is $2 m \times 2 m$, it follows from Eq. (116) that $\Delta \boldsymbol{U}_{k} \Delta \boldsymbol{u}_{k}^{T}$ is singular for all $k<2 m$. Hence the recursive procedure (113-115) cannot be used for $k<2 m$.

A suboptimal approach for determining an estimate $\hat{\boldsymbol{T}}_{k}$ of $\boldsymbol{T}$ is to replace $\boldsymbol{P}_{k}$ in Eqs. (113-115) by $\hat{\boldsymbol{P}}_{k}$, that is,

$$
\begin{gathered}
\boldsymbol{K}_{k+1}=\left(1+\Delta \boldsymbol{u}_{k+1}^{T} \hat{\boldsymbol{P}}_{k} \Delta \boldsymbol{u}_{k+1}\right)^{-1} \Delta \boldsymbol{u}_{k+1}^{T} \hat{\boldsymbol{P}}_{k} \\
\hat{\boldsymbol{T}}_{k+1}=\hat{\boldsymbol{T}}_{k}+\varepsilon_{k+1} \boldsymbol{K}_{k+1} \\
\hat{\boldsymbol{P}}_{k+1}=\hat{\boldsymbol{P}}_{k}\left(I-\Delta \boldsymbol{u}_{k+1} \boldsymbol{K}_{k+1}\right)
\end{gathered}
$$

where $\hat{\boldsymbol{P}}_{0}$ is positive definite but otherwise arbitrary. It follows from Eqs. (117-119) that $\hat{\boldsymbol{P}}_{k}$ is positive definite for all $k \geq 0$ and is given by

$$
\hat{\boldsymbol{P}}_{k}=\left(\hat{\boldsymbol{P}}_{0}^{-1}+\Delta \boldsymbol{U}_{k} \Delta \boldsymbol{U}_{k}^{T}\right)^{-1}
$$

Furthermore $\hat{\boldsymbol{T}}_{k}$ is given by

$$
\hat{\boldsymbol{T}}_{k}=\Delta \boldsymbol{Z}_{k} \Delta \boldsymbol{U}_{k}^{T}\left(\hat{\boldsymbol{P}}_{0}^{-1}+\Delta \boldsymbol{U}_{k} \Delta \boldsymbol{U}_{k}^{T}\right)^{-1}
$$

In this approach, because $\hat{\boldsymbol{P}}_{0}$ is positive definite the inverse in Eq. (121) always exists, and hence the recursive procedure can be used for all $k \geq 0$. The updated estimate $\hat{\boldsymbol{T}}_{k}$ is used at each control update step to calculate the control law $\boldsymbol{u}_{k+1}$, which is given by

$$
\boldsymbol{u}_{k+1}=-\hat{\boldsymbol{M}}_{k}\left(\boldsymbol{z}_{k}-\hat{\boldsymbol{T}}_{k} \boldsymbol{u}_{k}\right)
$$

where $\hat{\boldsymbol{M}}_{k}$ is defined by

$$
\hat{\boldsymbol{M}}_{k}=\left(\hat{\boldsymbol{T}}_{k}^{T} Q \hat{\boldsymbol{T}}_{k}+S^{T} \hat{\boldsymbol{T}}_{k}+\hat{\boldsymbol{T}}_{k}^{T} S+R\right)^{-1}\left(\hat{\boldsymbol{T}}_{k}^{T} \boldsymbol{Q}+\boldsymbol{S}^{T}\right)
$$

Assuming $\left(\Delta \boldsymbol{U}_{k} \Delta \boldsymbol{U}_{k}^{T}\right)^{-1}$ exists, it follows from Eq. (120) that if $\hat{\boldsymbol{P}}_{0} \rightarrow \infty$ then $\hat{\boldsymbol{P}}_{k} \rightarrow\left(\Delta \boldsymbol{U}_{k} \Delta \boldsymbol{U}_{k}^{T}\right)^{-1}$, and hence Eqs. (101) and (121) imply that $\hat{\boldsymbol{T}}_{k} \rightarrow \hat{\boldsymbol{T}}_{\mathrm{LS}_{k}}$.

A convergence analysis for adaptive $\mathrm{HHC}$ is provided in the Appendix.

\section{Illustrative Results}

A numerical study was performed to evaluate the use of the relaxation factor in highly nonlinear flight conditions, when the conventional invariant version of $\mathrm{HHC}$ is unable to reduce vibrations. Simulations were also performed where the initial identification of the sensitivity matrix $\boldsymbol{T}$ is poor, to judge controller response.

\section{Description of Simulation}

An aeroelastic code, described in Refs. 20 and 24, was used to perform simulations on a helicopter that resembles an MBB BO105; model parameters are listed in Table 1 . The helicopter is flying with an advance ratio of $\mu=0.15$, a flight condition where a large amount of BVI is experienced, inducing high levels of vibration and noise. Two flight conditions at the same advance ratio were considered: 1) a condition corresponding to 6-deg descending flight in heavy BVI, and 2) level flight where BVI effects are reduced. Most of the results presented are for the descending flight case where the degree of nonlinearity is larger.

Vibration reduction is achieved by means of a single partial-span actively controlled flap located at the trailing edge on each blade, ${ }^{20,24}$ as shown in Fig. 1. The control input vector $\boldsymbol{u}_{k}$ consists of sine and

Table 1 Elastic blade configuration

\begin{tabular}{lc}
\hline \hline Variable & Value \\
\hline \multicolumn{3}{c}{ Rotor data } & \\
$N_{b}$ & 4 \\
$\omega_{F 1}$ & 1.123 \\
$\omega_{L 1}$ & 0.732 \\
$\omega_{T 1}$ & 3.17 \\
$\theta_{t w}$ & $-8 \mathrm{deg}$ \\
$\gamma$ & 5.5 \\
Precone angle, deg & 2.5 \\
$c$ & $0.05498 L_{b}$ \\
$C_{\mathrm{do}}$ & 0.01 \\
$C_{\mathrm{mo}}$ & 0.0 \\
$a_{o}$ & $2 \pi$ \\
$\sigma$ & 0.07 \\
& \\
$C_{W}$ & Helicopter data \\
$X_{\mathrm{FA}}$ & 0.005 \\
$X_{\mathrm{FC}}$ & 0.0 \\
$\mu$ & 0.0 \\
$Z_{\mathrm{FA}}$ & 0.15 \\
$Z_{\mathrm{FC}}$ & 0.3 \\
\hline \hline
\end{tabular}




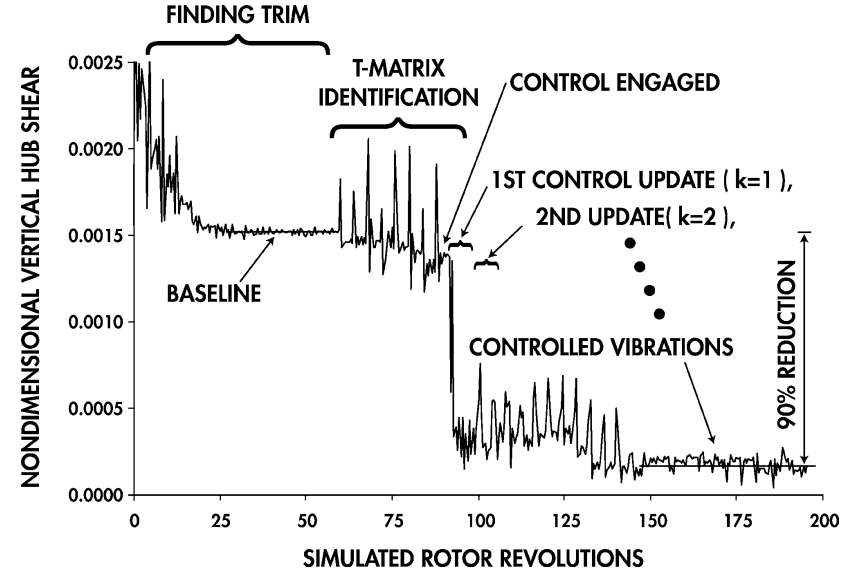

Fig. 4 Representative vibration reduction process in the aeroelastic simulation. cosine components of 2/rev, 3/rev, 4/rev, and 5/rev harmonics. The vector $z_{k}$ consists of the sine and cosine components of the six 4/rev hub shears and moments. Thus, $\boldsymbol{u}_{k}$ has length $2 m=8$, whereas $\boldsymbol{z}_{k}$ has length $2 p=12$.

The aeroelastic response code computes steady-state solutions over several revolutions; typically eight are used to reach a converged solution, and thus there are eight revolutions between controller updates $k$ and $k+1$. The HHC algorithm requires that a steady state be reached between control updates, as described earlier in this paper. To determine the effect of a poorly estimated $\boldsymbol{T}$ matrix on HHC, only one revolution is used in the identification process instead of the typical eight. This results in a $\boldsymbol{T}$ matrix with an error that does not strictly correspond to the additive or multiplicative error types used in convergence analysis described earlier in this paper. However, this error can represent real-time measurement error experienced on an actual helicopter.

Both invariant and adaptive HHC algorithms are considered. The rotor response is calculated by first finding the trim condition, then proceeding to system identification. The $\boldsymbol{T}$ matrix is identified before
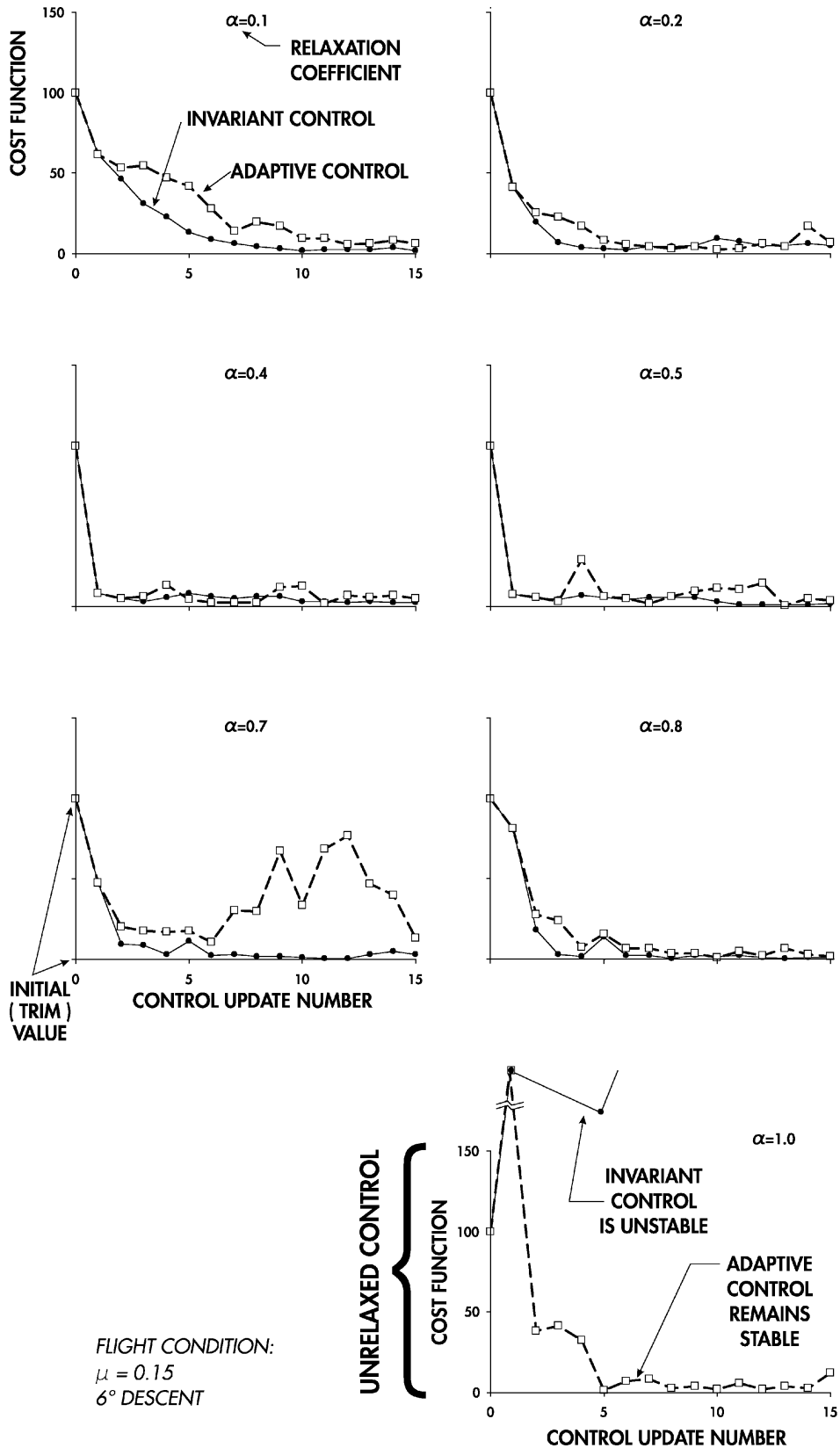
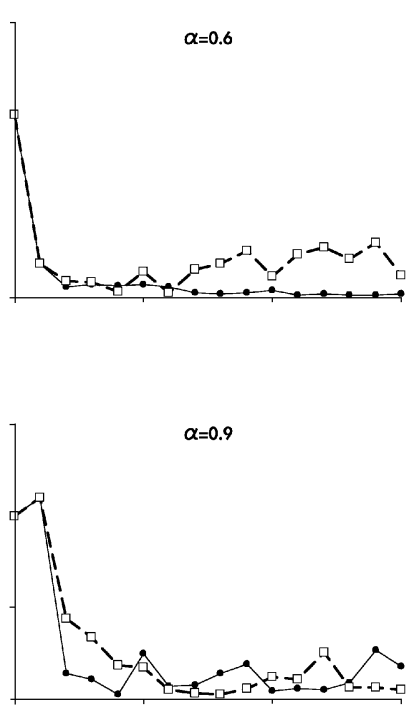

LEGEND:

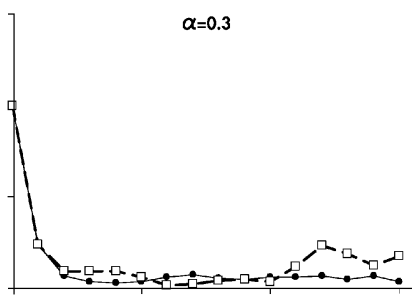

ADAPTIVE CONTROL $\square---\square$

INVARIANT CONTROL

Fig. 5 Comparison of adaptive and classical HHC algorithms when $T$ is well identified. 

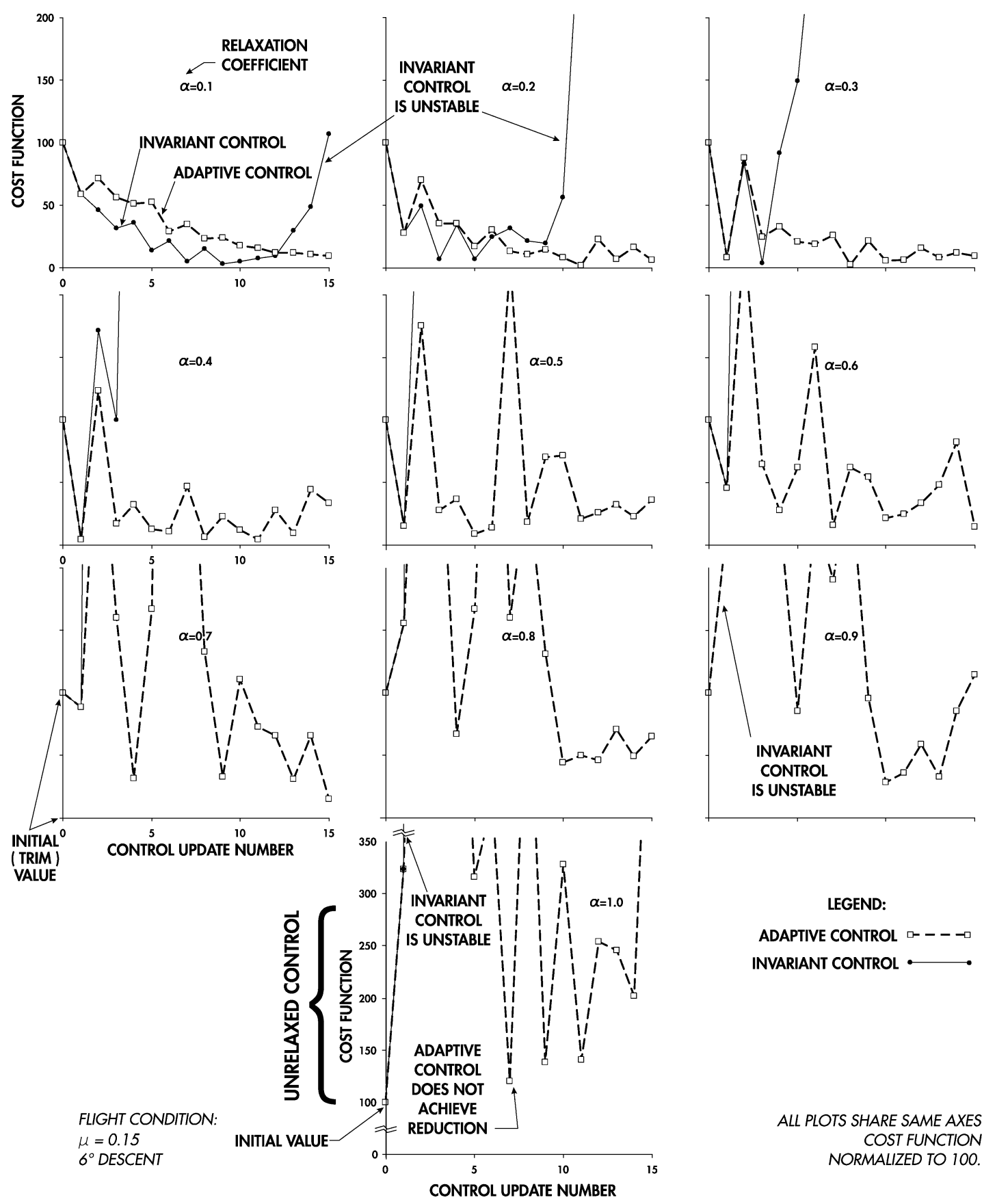

LEGEND:

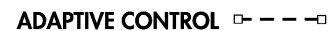

INVARIANT CONTROL

ALL PLOTS SHARE SAME AXES COST FUNCTION NORMALIZED TO 100.

Fig. 6 Comparison of adaptive and classical HHC algorithms when $T$ is poorly identified.

closed-loop control is engaged for both the invariant and adaptive $\mathrm{HHC}$ algorithms. Following trim and identification, closed-loop control is turned on, with updates between every eight rotor revolutions to allow the system to return to a steady state. The simulation is allowed to proceed for 10-25 control updates (values of $k$ ), and the cost function $J$ is tracked. A representative plot of the changes in the nondimensional 4/rev vertical hub shear during this process is shown in Fig. 4, for the level flight case.

The primary goal here is to quantify the effect of the relaxation factor on robustness. Four categories of simulations were performed: classical invariant HHC with both good and poor estimates of $\boldsymbol{T}$ and adaptive HHC with both good and poor estimates of $\boldsymbol{T}$. For each of these cases, the relaxation coefficient was varied from $\alpha=0.1$ to 1.0 (unrelaxed control), resulting in a total of 40 simulations. The relaxed adaptive variant is implemented with the same update rule as for the invariant case:
An analytic robustness and convergence analysis of relaxed adaptive HHC is not considered at this time. An exponential window ${ }^{2}$ is also implemented for adaptive control. The exponential window acts as a "forgetting factor," emphasizing the more current data:

$$
F_{f}=\gamma_{f}^{k}
$$

where $\gamma_{f}$ is the exponential window parameter varying $0<\gamma_{f}<1$, with $\gamma_{f}=0.8$ used in this study. This is implemented by modifying Eqs. (117-119) with Eq. (124):

$$
\begin{gathered}
\boldsymbol{K}_{k+1}=\left(\gamma_{f}^{k}+\Delta \boldsymbol{u}_{k+1}^{T} \hat{\boldsymbol{P}}_{k} \Delta \boldsymbol{u}_{k+1}\right)^{-1} \Delta \boldsymbol{u}_{k+1}^{T} \hat{\boldsymbol{P}}_{k} \\
\hat{\boldsymbol{T}}_{k+1}=\hat{\boldsymbol{T}}_{k}+\varepsilon_{k+1} \boldsymbol{K}_{k+1} \\
\hat{\boldsymbol{P}}_{k+1}=\hat{\boldsymbol{P}}_{k}\left[\left(1 / \gamma_{f}^{k}\right) I-\Delta \boldsymbol{u}_{k+1} \boldsymbol{K}_{k+1}\right]
\end{gathered}
$$




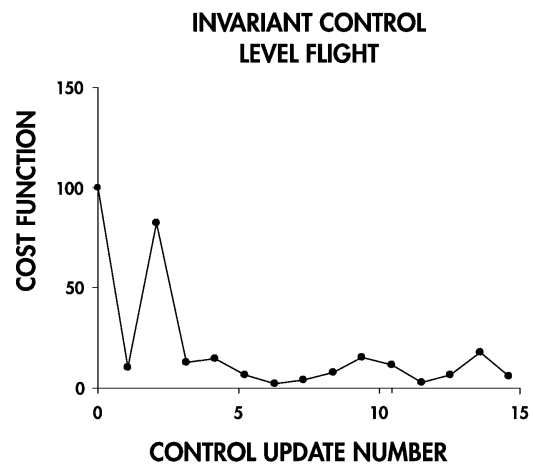

ALL PLOTS SHARE SAME AXES. COST FUNCTION NORMALIZED TO 100.
ADAPTIVE CONTROL LEVEL FLIGHT

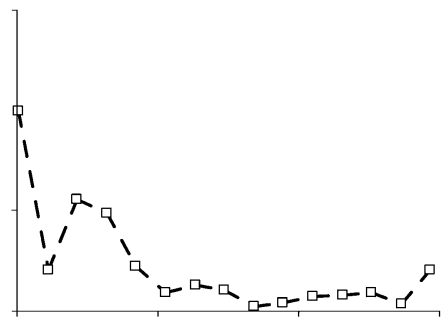

LEGEND:

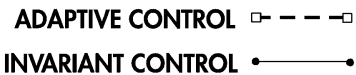

Fig. 7 Effect of flight condition on controller performance.

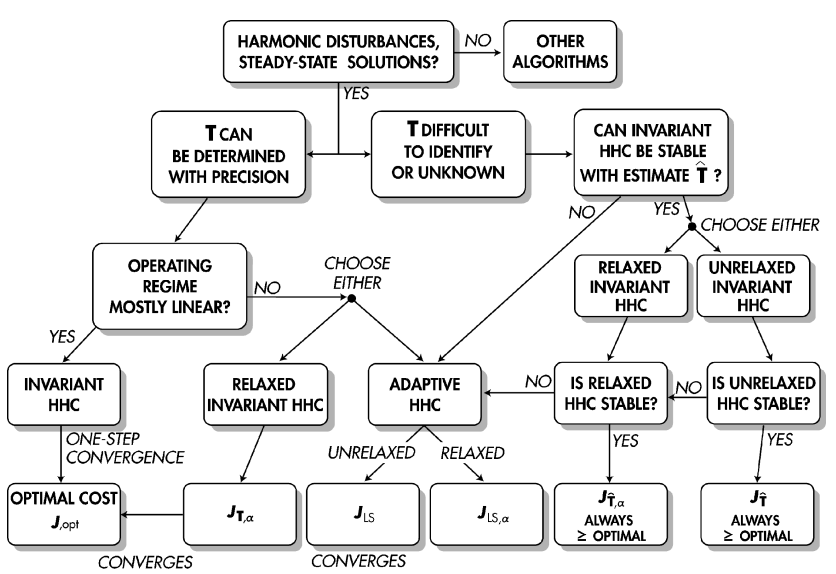

Fig. 8 Comparison of the variants of the HHC algorithm.

\section{Simulated Results}

The adaptive and invariant versions of the HHC algorithm are compared in Fig. 5 over the range of relaxation coefficients $\alpha=0.1$ to 1.0 for the descending flight case. When $\alpha=1.0$ (unrelaxed control), the invariant algorithm fails to converge to the optimal value of the objective function, probably because of the operating nonlinearities of the descending flight condition. However, the adaptive algorithm achieves a near-optimal value in only five control updates. As the relaxation factor $\alpha$ is reduced, the classical (invariant) algorithm displays consistently improving convergence to the desired minimum.

Figure 6 illustrates the effect of poor estimation of the $\boldsymbol{T}$ matrix using the procedure outlined earlier, also for the descending flight case. In this case, the invariant algorithm is never stable, not even with a relaxation coefficient of $\alpha=0.1$. The adaptive algorithm displays problems achieving good minimum solutions. In the unrelaxed adaptive case, no vibration reduction is achieved. This highlights the importance of achieving steady-state solutions between control updates. With the relaxation factor introduced at $\alpha=0.1$, the adaptive algorithm does approach the minimum in a stable, albeit slow, manner. Interestingly, for the unrelaxed invariant case Eq. (34) suggests that a small increase in the weighting $\boldsymbol{R}$ on flap deflections could make the algorithm stable.

The effect of the flight condition on controller stability is shown in Fig. 7. Two conditions are shown: 1) the 6-deg descent case discussed earlier and 2) a simplified level flight case. It is seen that the unrelaxed invariant HHC algorithm achieves good vibration reduction for the level flight case. The adaptive variant also performs well. These results confirm experimental observations ${ }^{5}$ on fixedgain HHC effectiveness.

\section{Conclusions}

This paper has summarized the basic features of the HHC algorithm that has been frequently used for active vibration reduction on helicopter rotors in forward flight. Figure 8 presents an overview of the different variants of the HHC algorithm, which facilitates a unified view of the various versions of the algorithm. From this figure, it is apparent that the relaxed, adaptive, or adaptive-relaxed versions of the algorithm are best suited to actual flight conditions, when identification is difficult and response might not be linear.

Some specific conclusions can be drawn from this paper:

1) The invariant HHC algorithm converges to the optimum value in a single control update in the ideal case.

2) The adaptive variant of the algorithm quickly recovers from poor initialization.

3) The new relaxed version of the algorithm was shown to have potentially beneficial effects on the performance of the algorithm.

4) Explicit stability conditions were developed for several variants of $\mathrm{HHC}$, and it was noted that changing control weighting, relaxation factor, or accuracy of identification could affect algorithm stability.

5) From the simulation studies, it is clear that even in cases where identification is poor and model nonlinearity is important, the adaptive algorithm combined with a well-chosen relaxation coefficient can be effective at reducing the objective function.

6) At flight conditions where the model has a linear input-output response, the simulations indicate that the unrelaxed invariant HHC algorithm can achieve reductions in the cost function.

This paper provides a sound theoretical basis for this widely used algorithm. Such a comprehensive foundation will be useful for further research on active vibration and noise reduction in rotorcraft.

\section{Appendix: Convergence Analysis of the Estimate $\hat{T}_{k}$}

Define $\Delta \boldsymbol{T}_{k}$ of size $2 p \times 2 m$ by

$$
\Delta \boldsymbol{T}_{k}=\hat{\boldsymbol{T}}_{k}-\boldsymbol{T}
$$

where $\hat{\boldsymbol{T}}_{k}$ is updated by using Eqs. (117-119). Next, define the function $\boldsymbol{V}(\Delta \boldsymbol{T}, \hat{\boldsymbol{P}})$ by

$$
\boldsymbol{V}(\Delta \boldsymbol{T}, \hat{\boldsymbol{P}})=\Delta \boldsymbol{T} \hat{\boldsymbol{P}}^{-1} \Delta \boldsymbol{T}^{T}
$$

and $\Delta \boldsymbol{V}_{k}$ by

$$
\Delta \boldsymbol{V}_{k}=\boldsymbol{V}\left(\Delta \boldsymbol{T}_{k+1}, \hat{\boldsymbol{P}}_{k+1}\right)-\boldsymbol{V}\left(\Delta \boldsymbol{T}_{k}, \hat{\boldsymbol{P}}_{k}\right)
$$

where $\hat{\boldsymbol{P}}_{k}$ is updated by using Eqs. (117-119) and $\hat{\boldsymbol{P}}_{0}$ is the positivedefinite matrix used to initialize Eq. (119). Subtracting $\boldsymbol{T}$ from both sides of Eq. (118) yields

$$
\Delta \boldsymbol{T}_{k+1}=\Delta \boldsymbol{T}_{k}+\varepsilon_{k+1} \boldsymbol{K}_{k+1}
$$


whereas substituting Eqs. (102) and (A4) into Eq. (A3) yields

$$
\begin{aligned}
\Delta \boldsymbol{V}_{k} & =\left(\Delta \boldsymbol{T}_{k}+\varepsilon_{k+1} \boldsymbol{K}_{k+1}\right)\left(\hat{\boldsymbol{P}}_{k}^{-1} \Delta \boldsymbol{u}_{k+1} \Delta \boldsymbol{u}_{k+1}^{T}\right) \\
& \times\left(\Delta \boldsymbol{T}_{k}+\varepsilon_{k+1} \boldsymbol{K}_{k+1}\right)^{T}-\Delta \boldsymbol{T}_{k} \hat{\boldsymbol{P}}_{k}^{-1} \Delta \boldsymbol{T}_{k}^{T} \\
& =\Delta \boldsymbol{T}_{k} \Delta \boldsymbol{u}_{k+1} \Delta \boldsymbol{u}_{k+1}^{T} \Delta \boldsymbol{T}_{k}^{T}+\Delta \boldsymbol{T}_{k} \Delta \boldsymbol{u}_{k+1} \Delta \boldsymbol{u}_{k+1}^{T} \boldsymbol{K}_{k+1}^{T} \varepsilon_{k+1}^{T} \\
& +\Delta \boldsymbol{T}_{k} \hat{\boldsymbol{P}}_{k}^{-1} \boldsymbol{K}_{k+1}^{T} \varepsilon_{k+1}^{T}+\varepsilon_{k+1} \boldsymbol{K}_{k+1} \hat{\boldsymbol{P}}_{k}^{-1} \Delta \boldsymbol{T}_{k}^{T} \\
& +\varepsilon_{k+1} \boldsymbol{K}_{k+1} \hat{\boldsymbol{P}}_{k}^{-1} \boldsymbol{K}_{k+1}^{T} \varepsilon_{k+1}^{T}+\varepsilon_{k+1} \boldsymbol{K}_{k+1} \Delta \boldsymbol{u}_{k+1} \Delta \boldsymbol{u}_{k+1}^{T} \Delta \boldsymbol{T}_{k}^{T} \\
& +\varepsilon_{k+1} \boldsymbol{K}_{k+1} \Delta \boldsymbol{u}_{k+1} \Delta \boldsymbol{u}_{k+1}^{T} \boldsymbol{K}_{k+1}^{T} \varepsilon_{k+1}^{T}
\end{aligned}
$$

By using Eq. (A1), $\varepsilon_{k+1}$ in Eq. (109) can be expressed as

$$
\varepsilon_{k+1}=-\Delta \boldsymbol{T}_{k} \Delta \boldsymbol{u}_{k+1}
$$

Substituting Eq. (A6) into Eq. (A5) yields

$$
\begin{aligned}
\Delta \boldsymbol{V}_{k} & =\varepsilon_{k+1}\left[1-\frac{2 \Delta \boldsymbol{u}_{k+1}^{T} \hat{\boldsymbol{P}}_{k} \Delta \boldsymbol{u}_{k+1}}{1+\Delta \boldsymbol{u}_{k+1}^{T} \hat{\boldsymbol{P}}_{k} \Delta \boldsymbol{u}_{k+1}}-\frac{2}{1+\Delta \boldsymbol{u}_{k+1}^{T} \hat{\boldsymbol{P}}_{k} \Delta \boldsymbol{u}_{k+1}}\right. \\
& \left.+\frac{\Delta \boldsymbol{u}_{k+1}^{T} \hat{\boldsymbol{P}}_{k} \Delta \boldsymbol{u}_{k+1}}{\left(1+\Delta \boldsymbol{u}_{k+1}^{T} \hat{\boldsymbol{P}}_{k} \Delta \boldsymbol{u}_{k+1}\right)^{2}}+\frac{\left(\Delta \boldsymbol{u}_{k+1}^{T} \hat{\boldsymbol{P}}_{k} \Delta \boldsymbol{u}_{k+1}\right)^{2}}{\left(1+\Delta \boldsymbol{u}_{k+1}^{T} \hat{\boldsymbol{P}}_{k} \Delta \boldsymbol{u}_{k+1}\right)^{2}}\right] \varepsilon_{k+1}^{T} \\
& =-\frac{\varepsilon_{k+1} \varepsilon_{k+1}^{T}}{1+\Delta \boldsymbol{u}_{k+1}^{T} \hat{\boldsymbol{P}}_{k} \Delta \boldsymbol{u}_{k+1}}
\end{aligned}
$$

and hence $\boldsymbol{V}\left(\Delta \boldsymbol{T}_{k}, \hat{\boldsymbol{P}}_{k}\right)$ is nonincreasing. Because $\boldsymbol{V}\left(\Delta \boldsymbol{T}_{k}, \hat{\boldsymbol{P}}_{k}\right) \geq 0$, it follows that $\lim _{k \rightarrow \infty} \boldsymbol{V}\left(\Delta \boldsymbol{T}_{k}, \hat{\boldsymbol{P}}_{k}\right)$ exists and is nonnegative. Hence,

$$
\lim _{k \rightarrow \infty}-\frac{\varepsilon_{k+1} \varepsilon_{k+1}^{T}}{1+\Delta \boldsymbol{u}_{k+1}^{T} \hat{\boldsymbol{P}}_{k} \Delta \boldsymbol{u}_{k+1}}=\lim _{k \rightarrow \infty} \Delta \boldsymbol{V}_{k}=0
$$

Next it is shown that $\Delta \boldsymbol{u}_{k}$ is bounded. Substituting Eq. (99) into Eq. (121) yields

$$
\hat{\boldsymbol{T}}_{k}=T \Delta \boldsymbol{U}_{k} \Delta \boldsymbol{U}_{k}^{T}\left(\hat{\boldsymbol{P}}_{0}^{-1}+\Delta \boldsymbol{U}_{k} \Delta \boldsymbol{U}_{k}^{T}\right)^{-1}
$$

Postmultiplying Eq. (A9) by $\hat{\boldsymbol{P}}_{0}^{-1}+\Delta \boldsymbol{U}_{k} \Delta \boldsymbol{U}_{k}^{T}$ yields

$$
\Delta \boldsymbol{T}_{k}=-\boldsymbol{T} \hat{\boldsymbol{P}}_{0}^{-1}\left(\hat{\boldsymbol{P}}_{0}^{-1}+\Delta \boldsymbol{U}_{k} \Delta \boldsymbol{U}_{k}^{T}\right)^{-1}
$$

Note that

$$
\begin{gathered}
\sigma_{\max }\left(\Delta \boldsymbol{T}_{k}\right) \leq \sigma_{\max }(\boldsymbol{T}) \sigma_{\max }\left(\hat{\boldsymbol{P}}_{0}^{-1}\right) \sigma_{\max }\left[\left(\hat{\boldsymbol{P}}_{0}^{-1}+\Delta \boldsymbol{U}_{k} \Delta \boldsymbol{U}_{k}^{T}\right)^{-1}\right] \\
\leq \frac{\sigma_{\max }(\boldsymbol{T})}{\sigma_{\min }\left(\hat{\boldsymbol{P}}_{0}\right) \sigma_{\min }\left(\hat{\boldsymbol{P}}_{0}^{-1}+\Delta \boldsymbol{U}_{k} \Delta \boldsymbol{U}_{k}^{T}\right)}
\end{gathered}
$$

if $\Delta \boldsymbol{U}_{k_{0}} \Delta \boldsymbol{U}_{k_{0}}^{T}$ is nonsingular, then for all $k \geq k_{0}$, Eq. (A11) implies that

$$
\sigma_{\max }\left(\Delta \boldsymbol{T}_{k}\right) \leq \frac{\sigma_{\max }(\boldsymbol{T})}{\sigma_{\min }\left(\hat{\boldsymbol{P}}_{0}\right) \sigma_{\min }\left(\Delta \boldsymbol{U}_{k_{0}} \Delta \boldsymbol{U}_{k_{0}}^{T}\right)}
$$

Hence, if $\hat{\boldsymbol{P}}_{0}$ is chosen to be sufficiently large, then $\sigma_{\max }\left(\Delta \boldsymbol{T}_{k}\right)$ can be made sufficiently small to satisfy the condition given by Eq. (34). For this case, Eq. (33) implies that, for all $k \geq k_{0}$,

$$
\sigma_{\max }\left(\hat{\boldsymbol{M}}_{k} \Delta \boldsymbol{T}_{k}\right)<1
$$

The state-space representation of the system dynamics with the control law, given by Eq. (122), is

$$
\left[\begin{array}{l}
\boldsymbol{z}_{k+1} \\
\boldsymbol{u}_{k+1}
\end{array}\right]=\hat{\boldsymbol{A}}_{k}\left[\begin{array}{l}
\boldsymbol{z}_{k} \\
\boldsymbol{u}_{k}
\end{array}\right]
$$

where $\hat{\boldsymbol{A}}_{k}$ is defined in Eq. (28) with $\hat{\boldsymbol{T}}$ and $\hat{\boldsymbol{M}}$ replaced by $\hat{\boldsymbol{T}}_{k}$ and $\hat{\boldsymbol{M}}_{k}$, respectively. Hence for all $k \geq k_{0}$

$$
\left[\begin{array}{l}
\boldsymbol{z}_{k} \\
\boldsymbol{u}_{k}
\end{array}\right]=\prod_{i=k_{0}}^{k} \hat{\boldsymbol{A}}_{i}\left[\begin{array}{l}
\boldsymbol{z}_{k_{0}} \\
\boldsymbol{u}_{k_{0}}
\end{array}\right]
$$

Note that $\prod_{i=k_{0}}^{k} \hat{\boldsymbol{A}}_{i}$ can be factored as

$$
\begin{aligned}
\prod_{i=k_{0}}^{k} \hat{\boldsymbol{A}}_{i} & =\left[\begin{array}{cc}
\boldsymbol{I}_{2 p} & \boldsymbol{T} \\
0 & \boldsymbol{I}_{2 m}
\end{array}\right] \\
\times & {\left[-\hat{\boldsymbol{M}}_{k_{0}}\left[I+\sum_{i=k_{0}}^{k-1}\left(\prod_{j=0}^{i} \Delta \boldsymbol{T}_{j} \hat{\boldsymbol{M}}_{j+1}\right)\right] \prod_{i=k_{0}}^{k}\left(\hat{\boldsymbol{M}}_{i} \Delta \boldsymbol{T}_{i}\right)\right] } \\
\times & {\left[\begin{array}{cc}
\boldsymbol{I}_{2 p} & -\boldsymbol{T} \\
0 & \boldsymbol{I}_{2 m}
\end{array}\right] }
\end{aligned}
$$

From Eq. (A13) it follows that

$$
\sigma_{\max }\left[\prod_{i=k_{0}}^{k}\left(\hat{\boldsymbol{M}}_{i} \Delta \boldsymbol{T}_{i}\right)\right] \leq \prod_{i=k_{0}}^{k}\left[\sigma_{\max }\left(\hat{\boldsymbol{M}}_{i} \Delta \boldsymbol{T}_{i}\right)\right]<1
$$

and from Eqs. (A15-A17) one obtains that $z_{k}$ and $\boldsymbol{u}_{k}$ are bounded. Hence, for all $k=0,1, \ldots$, with $\gamma>0$

$$
\left\|\boldsymbol{u}_{k}\right\|<\gamma
$$

and thus

$$
\left\|\boldsymbol{u}_{k}\right\|=\left\|\boldsymbol{u}_{k+1}-\boldsymbol{u}_{k}\right\|<2 \gamma
$$

From Eq. (102) it follows that $\hat{\boldsymbol{P}}_{k+1} \leq \hat{\boldsymbol{P}}_{k}$, which implies that

$$
\hat{\boldsymbol{P}}_{k} \leq \hat{\boldsymbol{P}}_{0}
$$

Hence, it follows from Eqs. (A19) and (A20) that

$$
1+\Delta \boldsymbol{u}_{k}^{T} \hat{\boldsymbol{P}}_{k} \Delta \boldsymbol{u}_{k} \leq 1+\lambda_{\max }\left(\hat{\boldsymbol{P}}_{0}\right) \Delta \boldsymbol{u}_{k}^{T} \Delta \boldsymbol{u}_{k} \leq 1+4 \gamma^{2} \lambda_{\max }\left(\hat{\boldsymbol{P}}_{0}\right)
$$

and thus Eq. (A9) implies that

$$
\lim _{k \rightarrow \infty} \varepsilon_{k}=0
$$

Taking the limit as $k \rightarrow \infty$ of Eq. (118) yields

$$
\lim _{k \rightarrow \infty}\left(\hat{\boldsymbol{T}}_{k+1}-\hat{\boldsymbol{T}}_{k}\right)=\lim _{k \rightarrow \infty} \frac{\varepsilon_{k+1} \Delta \boldsymbol{u}_{k+1}^{T} \hat{\boldsymbol{P}}_{k}}{1+\Delta \boldsymbol{u}_{k+1}^{T} \hat{\boldsymbol{P}}_{k} \Delta \boldsymbol{u}_{k+1}}
$$

From Eqs. (A19-A22) it follows that

$$
\lim _{k \rightarrow \infty} \frac{\varepsilon_{k+1} \Delta \boldsymbol{u}_{k+1}^{T} \hat{\boldsymbol{P}}_{k}}{1+\Delta \boldsymbol{u}_{k+1}^{T} \hat{\boldsymbol{P}}_{k} \Delta \boldsymbol{u}_{k+1}}=0
$$

and hence

$$
\lim _{k \rightarrow \infty}\left(\hat{\boldsymbol{T}}_{k+1}-\hat{\boldsymbol{T}}_{k}\right)=0
$$

Thus, $\left\{\hat{\boldsymbol{T}}_{k}\right\}$ is a Cauchy sequence, and hence $\hat{\boldsymbol{T}}_{k}$ converges. However, there is no guarantee that $\hat{\boldsymbol{T}}_{k}$ will converge to $\boldsymbol{T}$. In fact, it can be shown that there are certain choices of $\hat{\boldsymbol{P}}_{0}$ and $\hat{\boldsymbol{T}}_{0}$ such that $\hat{\boldsymbol{T}}_{k}$ will not converge to $\boldsymbol{T}$. For example, if $\boldsymbol{u}_{0}=0, \boldsymbol{S}=0$, and $\hat{\boldsymbol{T}}_{0}=0$, then Eq. (122) implies that $\boldsymbol{u}_{1}=0$. Hence, it follows from Eqs. (117) and (118) that $\hat{\boldsymbol{T}}_{k}=0$ for all $k \geq 0$. Conditions on $\hat{\boldsymbol{T}}_{0}$ and $\hat{\boldsymbol{P}}_{0}$ that guarantee convergence of $\hat{\boldsymbol{T}}_{k}$ to $\overline{\boldsymbol{T}}$ are not available. 


\section{Acknowledgments}

This research was supported in part by a Rackham Predoctoral Fellowship from the University of Michigan for D. Patt, and by Army Research Office Grant 02-1-0202 with G. Anderson as Grant Monitor. Partial support by the FXB Center for Rotary and Fixed Wing Air Vehicle Design is also gratefully acknowledged.

\section{References}

${ }^{1}$ Friedmann, P. P., and Millott, T. A., "Vibration Reduction in Rotorcraft Using Active Control: A Comparison of Various Approaches," Journal of Guidance, Control, and Dynamics, Vol. 18, No. 4, 1995, pp. 664-673.

${ }^{2}$ Johnson, W., "Self-Tuning Regulators for Multicyclic Control of Helicopter Vibrations," NASA TP 1996, May 1982.

${ }^{3}$ McHugh, F. J., and Shaw, J., "Helicopter Vibration Reduction with Higher Harmonic Blade Pitch," Journal of the American Helicopter Society, Vol. 23, No. 4, 1978, pp. 26-35.

${ }^{4}$ Shaw, J., and Albion, N., "Active Control of the Helicopter Rotor for Vibration Reduction," Journal of the American Helicopter Society, Vol. 26, No. 3, 1981, pp. 32-39.

${ }^{5}$ Shaw, J., Albion, N., Hanker, E. J., and Teal, R. S., "Higher Harmonic Control: Wind Tunnel Demonstration of Fully Effective Vibratory Hub Force Suppression," Journal of the American Helicopter Society, Vol. 34, No. 1, 1989, pp. 14-25.

${ }^{6}$ Hammond, C. E., "Wind Tunnel Results Showing Rotor Vibratory Loads Reduction Using Higher Harmonic Blade Pitch," Journal of the American Helicopter Society, Vol. 28, No. 1, 1983, pp. 10-15.

${ }^{7}$ Molusis, J. A., Hammond, C. E., and Cline, J. H., "A Unified Approach to the Optimal Design of Adaptive and Gain Scheduled Controllers to Achieve Minimum Helicopter Rotor Vibration," Journal of the American Helicopter Society, Vol. 28, No. 2, 1983, pp. 9-18.

${ }^{8}$ Molusis, J. A., "The Importance of Nonlinearity on the Higher Harmonic Control of Helicopter Vibration," Proceedings of the 39th Annual Forum of the American Helicopter Society (St. Louis, MO), American Helicopter Society, Alexandria, VA, 1983, pp. 624-647.

${ }^{9}$ Wood, E. R., Powers, R. W., Cline, J. H., and Hammond, C. E., "On Developing and Flight Testing a Higher Harmonic Control System," Journal of the American Helicopter Society, Vol. 30, No. 1, 1985, pp. 3-20.

${ }^{10}$ Straub, F. K., and Byrns, E. V., "Application of Higher Harmonic Blade Feathering on the OH-6A Helicopter for Vibration Reduction," NASA CR 4031, Dec. 1986

${ }^{11}$ Polychroniadis, M., "Generalized Higher Harmonic Control, Ten Years of Aerospatiale Experience," Proceedings of the Sixteenth European Rotorcraft Forum, Vol. 2, Glasgow, Scotland, U.K., 1990, pp. 3.7.2.1-3.7.2.9.
${ }^{12}$ Robinson, L. H., and Friedmann, P. P., "A Study of Fundamental Issues in Higher Harmonic Control Using Aeroelastic Simulation," Journal of the American Helicopter Society, Vol. 36, No. 2, 1991, pp. 32-43.

${ }^{13}$ Nygren, K. P., and Schrage, D. P., "Fixed-Gain Versus Adaptive Higher Harmonic Control Simulation," Journal of the American Helicopter Society, Vol. 34, No. 3, 1989, pp. 51-58.

${ }^{14}$ Jacklin, S. A., "Comparison of Five System Identification Algorithms for Rotorcraft Higher Harmonic Control,” NASA TP-1998-207687, May 1998.

${ }^{15}$ Hall, S. R., and Wereley, N. M., "Performance of Higher Harmonic Control Algorithms for Helicopter Vibration Reduction," Journal of Guidance, Control, and Dynamics, Vol. 16, No. 4, 1993, pp. 193-797.

${ }^{16}$ Chen, C. T., Linear System Theory and Design, Oxford Univ. Press, New York, 1998.

${ }^{17}$ Hall, S. R., and Wereley, N. M., "Linear Control Issues in the Higher Harmonic Control of Helicopter Vibrations," Proceedings of the 45th Annual Forum of the American Helicopter Society (Boston), American Helicopter Society, Alexandria, VA, 1989, pp. 955-959.

${ }^{18}$ Lovera, M., Colaneri, P., Malpica, C., and Celi, R., "Closed-Loop Aeromechanical Stability Analysis of $\mathrm{HHC}$ and IBC, with Application to a Hingeless Rotor Helicopter," Proceedings of the 29th European Rotorcraft Forum, 2003, pp. 43.1-43.14.

${ }^{19}$ Knospe, C. R., Hope, R. W., Tamer, S. M., and Fedigan, S. J., "Robustness of Adaptive Unbalance Control of Rotors with Magnetic Bearings," Journal of Vibration and Control, Vol. 2, No. 1, 1996, pp. 32-53.

${ }^{20}$ Patt, D., Liu, L., and Friedmann, P. P., "Rotorcraft Vibration Reduction and Noise Prediction Using a Unified Aeroelastic Response Simulation," Journal of the American Helicopter Society, Vol. 50, No. 1, 2005 , pp. 95-106.

${ }^{21}$ Depailler, G., "Alleviation of Dynamic Stall Induced Vibrations on Helicopter Rotors Using Actively Controlled Flaps," Ph.D. Dissertation, Dept. of Aerospace Engineering, Univ. of Michigan, Ann Arbor, MI, 2002.

${ }^{22}$ Cribbs, R., and Friedmann, P. P., "Actuator Saturation and Its Influence on Vibration Reduction by Actively Controlled Flaps," Proceedings of the 42nd AIAA/ASME/ASCE/AHS/ACS Structures, Structural Dynamics and Materials Conference, AIAA, Reston, VA, 2001.

${ }^{23}$ Hong, J., and Bernstein, D. S., "Bode Integral Constants, Collocation, and Spillover in Active Noise and Vibration Control," IEEE Transactions on Control System Technology, Vol. 6, No. 3, 1998, pp. 111-120.

${ }^{24}$ Patt, D., "Simultaneous Noise and Vibration Reduction in Rotorcraft Using Actively Controlled Flaps and Including Performance Considerations," Ph.D. Dissertation, Dept. of Aerospace Engineering, Univ. of Michigan, Ann Arbor, MI, 2004.

${ }^{25}$ Astrom, K. J., and Wittenmark, B., Adaptive Control, Addison Wesley Longman, Reading, MA, 1995. 